\title{
Financial Hedging and Optimal Procurement Policies under Correlated Price and Demand
}

\author{
Ankur Goel \\ Analytics and Portfolio Management, PNC Financial Services, Pittsburgh, Pennsylvania 15222, USA, ankur.goel@pnc.com
}

Fehmi Tanrisever

Faculty of Business Administration, Bilkent University, Bilkent, 06800 Ankara, Turkey, tanrisever@bilkent.edu.tr

\begin{abstract}
W e consider a firm that procures an input commodity to produce an output commodity to sell to the end retailer. $\checkmark$ The retailer's demand for the output commodity is negatively correlated with the price of the output commodity. The firm can sell the output commodity to the retailer through a spot, forward or an index-based contract. Input and output commodity prices are also correlated and follow a joint stochastic price process. The firm maximizes shareholder value by jointly determining optimal procurement and hedging policies. We show that partial hedging dominates both perfect hedging and no-hedging when input price, output price, and demand are correlated. We characterize the optimal financial hedging and procurement policies as a function of the term structure of the commodity prices, the correlation between the input and output prices, and the firm's operating characteristics. In addition, our analysis illustrates that hedging is most beneficial when output price volatility is high and input price volatility is low. Our model is tested on futures price data for corn and ethanol from the Chicago Mercantile Exchange.
\end{abstract}

Key words: integrated risk management; financial hedging; inventory management; yield uncertainty; myopic optima History: Received: January 2014; Accepted: March 2017 by Panos Kouvelis, after 3 revisions.

\section{Introduction}

Commodity price fluctuations create challenges for commodity processors in determining production, procurement, and risk-mitigation strategies. The impact of price risk is more profound on processors that have limited market power to pass a price increase in raw material to end customers. Many firms, including agricultural processors, steel manufacturers, and energy producers, are susceptible to the commodity price risk because both their input and output prices are market determined. The effect of commodity price fluctuations is twofold; it creates uncertainty in the margins, and affects demand if price and demand of the output product are correlated. In this regard, financial hedging ${ }^{1}$ can play a pivotal role in mitigating price risk and maximizing firm value. In addition to price risk, commodity processors also face demand risk, and optimize their operating policies to manage this risk. Since price and demand for various commodities are correlated, this results in an interaction between hedging and operating policies. In this study, we jointly optimize the financial hedging and operating decisions of a commodity

*This research was conducted when the author was a faculty at the Case Western Reserve University. processor in a multi-period model when commodity demand and price are correlated. We characterize the optimal financial hedging and inventory policies as a function of the term structure of the commodity prices, the correlation between the input and output prices, and the firm's operating characteristics. We show that partial hedging dominates perfect hedging ${ }^{2}$ for a firm when input and output commodity prices are positively correlated.

It is well established that in the absence of market frictions, corporate-level risk management is a valueneutral proposition, and operating and financial hedging decisions are separable (Modigliani and Miller 1958). Financial theory explains the use of financial derivatives through capital market imperfections (e.g., transaction costs, information asymmetries, and taxes) and agency problems (Froot et al. 1993, Jin and Jorion 2006, Smith and Stulz 1985). In this study, we consider a publicly traded commodity processor that operates to maximize shareholder value, and experiences a correlated demand with the price of its output commodity due to logistical frictions. These frictions result in the breakdown of the Modigliani and Miller (1958) framework and requires the joint optimization of hedging and operating policies. A negative correlation between demand and price results in the concavity of the objective function, 
which creates an incentive to reduce price volatility, and hence, a need to develop and integrate an optimal hedging policy with inventory decisions.

This research is motivated by the operations of an ethanol producer that procures corn in the spot market to produce ethanol to sell to the local retailer. The retailer's demand for ethanol is negatively correlated with the price of ethanol. Our firm, the ethanol producer, contracts with the retailer to sell ethanol using spot, forward or index-based contracts. The firm is a price taker, where corn and ethanol prices are correlated and follow a joint stochastic process. In each period, the firm determines the procurement and processing policies for corn and the hedging policy for ethanol sales. Excess inventory is carried over into the next period, and excess demand is backlogged. We show that the expected base stock policy is optimal under yield uncertainty, and it is characterized as a function of the term structure of the futures prices. When there is no yield uncertainty, we establish the conditions for the optimality of the myopic policy.

Renewable energy is a strategic issue in the United States (US) and in many other countries around the globe. In this regard, ethanol is considered a partial substitute for gasoline, reducing reliance on fossil fuels. In 2016, according to the US Department of Energy, the US is expected to process 5.28 billion bushels of corn, generating a record 14.54 billion gallons of ethanol. Ethanol producers buy and process corn to produce and sell ethanol to downstream retailers (jobbers). ${ }^{3}$ Ethanol is mixed with gasoline by the jobber in accordance with environmental regulations and the economics of the process. The retailer (the ethanol user) optimizes its gasoline blend based on ethanol prices and the chemical properties of the gasoline when it is mixed with ethanol. Maintaining state regulations, the retailer blends more ethanol as ethanol prices decrease and reduces the ethanol content of its product as prices increase. This situation leads to a negative correlation between the retailer's demand and the commodity price. ${ }^{4}$ Due to the correlation of demand and price, under high price realization, the ethanol producer observes a low demand for its output commodity and may not be able to sell all of its inventory to local retailers, and also may not clear the remaining inventory in the exchange market due to logistical frictions. This mismatch in production and demand of ethanol results in firms carrying excess inventory into subsequent periods. According to the US Energy Information Administration, there were about 20.9 million barrels of ethanol inventory on $8 / 26 / 2016$. This situation is consistent with a classic paper on the behavior of commodity prices by Deaton and Laroque (1992), according to which, in a multi-period setting, the optimal price does not necessarily clear the market and a firm carries positive inventory into the subsequent period. In this study, we model the negative correlation between ethanol price and demand, as well as the inventory dynamics in a multi-period framework.

Over the past few years, the effect of fossil-fuel supply chains on the environment has become a central issue in determining environmental policy across many countries. Environmental concerns over methyl tertiary butyl ether (MTBE), a substance blended with gasoline to raise the octane number, resulted in the US Environmental Protection Agency banning the substance in 2006. Ethanol now replaces MTBE as a means to improve the octane performance of gasoline. This change had a substantial impact on the economics of growing corn: ethanol production now represents the highest use of corn in the US, followed by its use for feed. As ethanol production consumes the largest portion of the corn supply, the prices of ethanol and corn have begun to affect each other. As a result, we model corn and ethanol prices with a mean-reverting correlated stochastic process. Furthermore, the conversion process of corn into ethanol is subject to yield uncertainty, and therefore, we also incorporate yield uncertainty when determining optimal procurement and hedging policies.

Another important issue in the supply chain of ethanol is its distribution cost. Ethanol has an affinity for water, rendering it unsuitable for transporting through pipelines. At present, the only possible modes of transportation for ethanol are trucks and trains, which result in transportation costs almost 10 times higher for ethanol compared to gasoline (Wakeley et al. 2009). This cost factor limits the economic feasibility of transporting ethanol over long distances. According to Wakeley et al. (2009), "Long-distance transport of ethanol to the end user can negate ethanol's potential economic and environmental benefits relative to gasoline." Therefore, the ethanol producer in our model prefers to carry excess inventory into subsequent periods rather than shipping this inventory to an end user outside the local market. These circumstances entail that the price elasticity of ethanol at the retailer's end is transferred to the ethanol producer due to the inability to sell excess inventory outside the local market because of high transportation costs.

The ethanol processor procures corn in the spot market and produces ethanol to sell in the local market. The price of ethanol in the local market is perfectly correlated with the price of ethanol on the Chicago Mercantile Exchange (CME). Ethanol producers sell ethanol to jobbers through a variety of contracts, including spot, forward, and index-based agreements (Dahlgran 2010, Franken and Parcell 2003). In our model, we propose an index-based contingent contract whose price and volume are determined as a function of spot and futures prices. The 
sales price of the contract is a weighted function of the futures price observed today for the contract that matures in the next period, and of the spot price to be observed in the next period. The expected price of this contract is always equal to the futures price; however, the weight on the futures contract vs. the spot contract can change the price volatility. Since this index contract reduces price volatility compared to the pure spot contract, we refer to this case as financial hedging. In particular, we show that for an ethanol producer, an index-based contract performs better than either spot or forward contracts, and we dynamically optimize the contract in each period.

Our contributions to the literature are as follows: (1) We show that partial hedging dominates perfect hedging and no-hedging for a publicly traded firm when input and output prices are positively correlated, and demand is negatively correlated with output price. (2) We identify three sufficient conditions under which a myopic policy is optimal for a pricetaker firm when output price and demand are negatively correlated. (3) We characterize the optimal policy for inventory procurement and the policy for hedging the sales of the output commodity as a function of the term structure of the futures prices, and show that the expected base stock policy is optimal under yield uncertainty. (4) Our numerical analysis shows that the value of hedging increases with output price volatility, but decreases with input price volatility. We also observe that yield uncertainty has a nonmonotone effect on the value of hedging. Our model is motivated by ethanol processing but it can be applied to many other commodity-processing scenarios, such as steel, wheat, and chemicals. We test our model on the futures price data obtained from the CME for corn and ethanol contracts traded from 4/1/ 2005 to $12 / 31 / 2011$.

\section{Literature Review}

Our study is related to the literature on commodity processing, as discussed in section 2.1. In our study, we bridge the gap between commodity processing and financial hedging in a multi-period framework. We discuss in detail the literature on financial hedging in operations and finance in sections 2.2 and 2.3, respectively. Agricultural commodity processors are constantly confronted with yield uncertainty in the challenge of matching supply and demand. In this context, in section 2.4, we discuss how our research relates to the literature on managing yield uncertainty by commodity processors.

\subsection{Commodity Processing}

There is a growing body of research on commodity processing and trading in the operations literature.
For example, Secomandi (2010) evaluates the value of storage for natural gas in the presence of operational constraints. Berling and Martinez-de Albeniz (2011) develop operating policies for commodity processors under stochastic price and demand. Goel and Gutierrez $(2009,2011)$ postulate the significance of dynamically updating operating policies in the presence of stochastically evolving convenience yield. Devalkar et al. (2011) obtain optimal commodity processing and storage decisions under capacity constraints. Similar capacity and risk management problems for agricultural commodities are addressed by Boyabatli et al. (2016) and Noparumpa et al. (2015). More recently, Devalkar et al. (2016) consider commodity processing and risk management in partially complete markets in the presence of financial distress costs.

Our study is also closely related to Plambeck and Taylor (2011, 2013), who consider the dynamics between input and output prices for a commodity processor in the absence of financial hedging. Plambeck and Taylor (2011) explore the value of operational flexibility, and show that the value of feedstock-intensity flexibility decreases with variability in feedstock cost or output price. Plambeck and Taylor (2013) study the trade-off between input efficiency and capacity efficiency, and conclude that the flexibility to adjust between these two types of efficiencies decreases with variability in input and output prices if the expected margin is thin. Our study contributes to the literature by (1) dynamically integrating financial hedging with operating decisions in a multi-period model, (2) considering the stochastic dynamics of both input and output prices, as well as the associated effect of correlation on hedging decisions, and (3) exploring the effect of yield uncertainty on hedging and operating decisions.

\subsection{Hedging Under Utility/Profit Maximization}

In the economics literature, Rolfo (1980) derives an optimal futures hedging strategy under both price and production uncertainties in a mean-variance framework. He shows that the optimal hedge ratio is equal to one in the absence of production risk, and is less than one in the presence of production risk. A similar result is later provided under a continuoustime model by Ho (1984), under a constant absolute risk-aversion (CARA) utility function of consumption. In contrast to Rolfo (1980) and Ho (1984), we show that even if there is no production risk, the optimal hedge ratio is less than one for a value-maximizing firm when input and output prices are positively correlated, and demand is negatively correlated with the output price.

Recently, financial hedging has received growing attention in the operations management literature. 
In the context of risk-averse decision makers, Gaur and Seshadri (2005) address the problem of hedging inventory risk when demand is correlated with the price of a financial asset. Similarly, Chen et al. (2007) show how to hedge operational risk through financial instruments in a partially complete market in a multi-period model. Dong and Liu (2007) derive a bilateral forward contract in a Nash bargaining framework, and justify its prevalence due to the hedging benefits in spite of the presence of a liquid spot market under a utility-maximization framework. Again in a risk-averse setting, Ding et al. (2007) show how a multi-national firm can use a real option to partially hedge against demand uncertainty, and use financial options on the currency exchange rate to hedge against currency risk. There is also a stream of research showing the relevance of risk management for risk-neutral decision makers. For example, Caldentey and Haugh (2009) explore the value of flexible supply contracts in conjunction with financial hedging, and Turcic et al. (2015) examine the value of hedging input costs in a decentralized supply chain with risk-neutral agents. Our study differs from the above papers in the following respects: (1) Our model is cast in a value-maximization framework, which is appropriate for well-diversified, publicly traded firms. (2) We integrate the dynamics of both input and output commodity prices into the firm's operating and hedging decisions. (3) We examine hedging in a multi-period model in conjunction with inventory dynamics. Next, we briefly discuss the finance literature on value maximization and financial hedging.

\subsection{Hedging Under Value Maximization}

Since the seminal paper of Modigliani and Miller (1958), it is now well known that trading financial derivatives is a value-neutral proposition for a firm under perfect capital markets. Financial theory explains the use of financial derivatives, in practice, through capital market imperfections (e.g., transaction costs, information asymmetries, and taxes) and agency problems (Froot et al. 1993, Jin and Jorion 2006, Smith and Stulz 1985). Financial hedging may also create value when risk-averse agents that contract with the firm cannot fully diversify their claims (Bessembinder 1991). In a stylized single-period setting, Froot et al. (1993) investigate an investment and hedging problem in the presence of costly external funds. They show that positive correlation between the availability of investment opportunities and the supply of internal cash flows creates a natural hedge; and hence, the firm underhedges in the financial market. Although this finding is similar to our underhedging result, our analysis and findings are different in the following respects: (1) We establish our results in the presence of logistical frictions, and they are driven by the correlation between the input and output prices and the output price and demand. (2) Our model incorporates inventory and price dynamics into the firm's hedging plan, and clearly delineates the role of demand, and holding and shortage costs. In addition, input and output prices as well as convenience yield are essential ingredients of the optimal policy. (3) Our hedge ratio is nonlinear in the correlation term due to the inventory and price dynamics in our model. Overall, we provide a dynamic hedging policy that can be easily implemented. Similar to the finance literature, in this study, we explore the value of hedging under a value-maximization framework.

\subsection{Yield Uncertainty}

The economics literature has typically considered the impact of yield uncertainty in a single-period setup cast in a utility-maximization framework. Rolfo (1980) shows that the optimal hedge ratio is not equal to one in the presence of yield uncertainty when individual preferences are represented either by logarithmic or quadratic utility functions. Losq (1982) extends the results of Rolfo (1980) to a general utilitymaximization framework and shows that when yield and price are independent, the firm should hedge less than the expected output, provided that the utility function shows decreasing absolute risk aversion. Moschini and Lapan $(1992,1995)$ explore the effect of correlation among yield, price, and basis risks on the optimal hedge ratio when agents' preferences are of a CARA type and in a mean-variance framework. Our paper focuses on the effect of yield uncertainty on hedging, and is cast in the value framework of finance. We also consider a multi-period model that closely captures the inventory dynamics of the problem.

In the operations literature, the structure of optimal operating policies has been well studied under yield uncertainty. Henig and Gerchak (1990) show that under yield uncertainty, order-up-to policies are not optimal in a periodic-review inventory model. More recently, Sobel and Babich (2012) prove the optimality of myopic policies with an order-up-to structure in a multi-echelon model with an autoregressive demand, under the assumption that yield uncertainty is independent of the lot size. In our model, we show that under the stochastically proportional yield model an expected base-stock policy is optimal. We also contribute to the literature on yield uncertainty in operations management by introducing financial hedging. To the best of our knowledge, this aspect has never been explored in this literature. 


\section{Commodity Processing with Hedging}

In this section, we present the mathematical model in section 3.1, then we characterize the optimal policy and determine the value of hedging in section 3.2. We first assume deterministic yield to understand the dynamics between commodity processing and financial hedging, and in section 4 we discuss the effect of yield uncertainty.

\subsection{Mathematical Model}

We consider a commodity processor that procures an input commodity to process and sell it to a retailer whose demand is negatively correlated with the price of the processed output commodity. We assume that

Assumption 1. The firm is a price taker for both input and output commodities.

Assumption 2. Both input and output commodities are traded on an organized exchange that offers no arbitrage opportunities.

Assumption 3. Without loss of generality, the processing of the output commodity has a lead time of one period.

Assumption 4. Excess demand is backlogged.

Assumption 5. The contract design between the firms is credible (see, e.g., Boyabatli et al. 2011, Kouvelis et al. 2013). Credibility of the contracts are ensured through collateral mechanisms similar to forward contracts.

Assumption 6. It is cheaper to hold inventory in the upper echelon.

In each period, the commodity processor (1) observes the spot prices of the input and output commodities and the inventory of the output commodity, and (2) determines how much input commodity to procure and process, as well as determining the hedging policy for the sales of the output commodity.

We respectively denote the spot prices of input and output commodities as $s_{t}$ and $p_{t}$, where $\left(s_{t}, p_{t}\right) \in \mathcal{P}_{t}$, and $\mathcal{P}_{t}$ contains all the information related to the state of the economy, demand and supply dynamics, and developing new technologies. The evolution of commodity prices follows a mean reverting process of the Ornstein-Uhlenbeck type, as outlined in the finance literature (see, e.g., Schwartz 1997, Schwartz and Smith 2000). This stochastic price process offers no risk-free arbitrage opportunities, such that $\mathcal{P}_{t}$ evolves under a risk-neutral measure where the futures price of the input commodity $i$ at time $t$, for delivery at time $t+1$, is given by $f_{t, t+1}^{i}=E_{\mathcal{P} \mid \mathcal{P}_{t}}^{\mathbb{Q}}\left[s_{t+1}\right]$. Similarly, the futures price of the output commodity $o$ at time $t$, for delivery at time $t+1$, is given by $f_{t, t+1}^{o}=E_{\mathcal{P} \mid \mathcal{P}_{t}}^{\mathbb{Q}}\left[p_{t+1}\right]$. The superscript $\mathbb{Q}$ denotes that the expectation is taken under the risk-neutral measure. Future cash flows evaluated under a risk-neutral measure are discounted at a risk-free rate $r_{f}$, such that the discount rate $\beta=\mathrm{e}^{-r_{f} \Delta t}$, where $\Delta t=1$. For notational brevity we denote the one-period-ahead futures prices simply by $f_{t}^{o}$ and $f_{t}^{i}$ for the output and input commodities, respectively.

Assumption 1 holds when the monopoly price in the local market, $s^{m}$, is larger than the spot price plus the logistical costs, $\lambda_{t}$, to access the exchange market. This is a reasonable condition, since it is well established in the economics literature that monopoly prices are higher than competitive market prices (Bresnahan 1982). The firm of our interest, the ethanol producer, is geographically located at a distance, such that it costs $\lambda_{t}$ to transport the output commodity to the exchange, where it is traded at price $p_{t}$. Our firm's customer is located locally, such that transportation costs between the two are negligible. If our firm optimizes the price in the local market then it can charge the monopoly price $s^{m}$ to its customers. However, since $s^{m} \geq p_{t}+\lambda_{t}$, it is profitable for the customer to procure the commodity from the exchange if the producer offers the monopoly price. Therefore, the maximum price our firm can charge is determined by the cost of the customer's outside option, $p_{t}+\lambda_{t}$. On the other hand, since the revenues are concave, there is no economic reason for charging a price below $p_{t}+\lambda_{t}$. As a result, the equilibrium spot price in the local market is $p_{t}+\lambda_{t}$.

It is common for commodity processors to sell an output commodity through spot and forward agreements. Also, in practice, there are index-based price contracts that are a combination of spot and futures prices. We define $W_{t}\left(b_{t}, p_{t+1}\right)=b_{t} f_{t}^{o}+\left(1-b_{t}\right)$ $p_{t+1}+\lambda_{t+1}$ as the index price that the customer agrees at time $t$ to pay at time $t+1$, where $b_{t} \in[0,1]$ is the hedging decision and $p_{t+1}$ is the realized price of the output commodity at time $t+1$. Simultaneously, at time $t$, the buyer commits to the quantity, $d_{t+1}=A_{t+1}-\gamma W_{t}\left(b_{t}, p_{t+1}\right)$, as a function of the index price $W_{t}\left(b_{t}, p_{t+1}\right)$, where $A_{t+1}$ is the maximum possible demand of the retailer (also called the market size) and $\gamma$ is the retailer's demand elasticity with respect to price (see, e.g., Inderfurth et al. 2014, Kazaz 2004). This contract is agreed upon at time $t$, but its value is realized at time $t+1$, after the realization of the spot price of the output commodity. That is, the contract between the processor and the retailer specifies the price and quantity $\left(W_{t}\left(b_{t}, p_{t+1}\right), d_{t+1}\right)$ in a contingent manner (see, e.g., Samuelson 1986, Bazerman and Gillespie 1998, and Biyalogorsky and Gerstner 2004 on contingent contracts). In summary, the processor and the 
retailer commit to a menu of price and quantity contracts at time $t$ to be delivered/realized at time $t+1$. Hence, there is a one-to-one mapping between the price and the quantity through the menu of contracts. The retailer is in a binding contract to get the quantity as agreed upon through the menu of contracts at time $t$, and does not have the leeway to adjust the quantity after the realization of the price at time $t+1$.

If the firm decides to completely hedge the price risk, then it chooses $b_{t}=1$, such that $W_{t}\left(b_{t}, p_{t+1}\right)=f_{t}^{o}+\lambda_{t+1}$. On the other hand, if the firm decides not to hedge, then $b_{t}=0$, such that $W_{t}\left(b_{t}, p_{t+1}\right)=p_{t+1}+\lambda_{t+1}$. As a result, the expected price is always $f_{t}^{o}+\lambda_{t+1}$, irrespective of the hedging policy. The hedging decision does not influence the expected price of the contract, but controls the variance of the price. Constraint $0 \leq b_{t} \leq 1$ ensures that the price of the index contract is always positive. We define the revenue function as a function of the index price, such that $\tilde{R}_{t}\left(b_{t}, p_{t+1}\right)=\left[A_{t+1}-\gamma W_{t}\left(b_{t}, p_{t+1}\right)\right]$ $W_{t}\left(b_{t}, p_{t+1}\right)$ for the realized spot price, $p_{t+1}$, and the hedging policy $b_{t}$.

In our model, the retailer, unlike the commodity processor, is a relatively small company that wants to avoid risk because of capital market frictions. We do not explicitly model these frictions, but costs such as bankruptcy or financial distress motivate the firm to avoid risk in the market (cf. Chod et al. 2010). We assume that these costs are high enough to compensate the retailer for the reduction in profits resulting from hedging the risk. Therefore, the retailer prefers to hedge the price risk through an index-based contract with the processing firm. The retailer also has access to the exchange market. However, procurement from the exchange is subject to a number of disadvantages, including basis risk. First of all, there is an inherent variability associated with the quality of the commodity supplied from the exchange. Resolving quality- and delivery-related issues is also harder when procuring from a distant exchange compared to a local firm. In addition, exchanges trade contracts with standard delivery dates; for example, the CME delivers wheat in March, May, July, September, and December only. If the procurement cycle of the retailer does not match the delivery cycle of the exchange, this results in basis risk which creates motivation to procure from the local producer. Due to the above disadvantages of buying from the exchange, the retailer prefers buying from a local processor. Nevertheless, anticipating these benefits, the processor may charge its customer a premium over the exchange market price. Indeed, this premium is a part of the transaction cost $\lambda_{t}$ in our model.

For expositional purposes, we consider that the input commodity is procured from the spot market. It is also possible for the firm to procure input commodity through forward contracts, but since procurement costs are linear in price and there are no financial or logistical frictions at the procurement end, forward procurement of input commodity is value-neutral. See Appendix B for a detailed proof of this result.

We denote $x_{t}$ as the current inventory of the output commodity, which can be used to satisfy customer demand. If the current inventory of the output commodity is insufficient to satisfy demand, then the firm incurs a backlog cost of $r$ per unit per period; on the other hand, if the demand is less than the current inventory, then the firm incurs a holding cost of $h$ per unit per period. We assume that excess demand is backlogged, and the holding cost is small relative to the sales price, such that it is economical for the firm to carry inventory rather than to reduce the price. One unit of input converts to $a$ units of output. We define $h^{\prime}$ as the holding cost per unit of input commodity, and from Assumption 6 we have $h>h^{\prime} / a$, which is in accordance with the multi-echelon inventory literature. In each period, the commodity processor observes the inventory of the output product $x_{t}$, the spot price for the input commodity $s_{t}$, and the spot price for the output commodity $p_{t}$, and then jointly determines the echelon stock $z_{t}$ and the hedging amount $b_{t}$ of the output commodity. Given the echelon stock $z_{t}$, the actual input commodity bought in the spot market to be processed is determined by $\left(z_{t}-x_{t}\right) / a$. The unit processing cost is denoted by $c_{t}$.

The objective of the firm is to maximize shareholder value in the presence of input and output commodity price risks. As suggested by Seppi (2002), we use the risk-neutral measure $\mathbb{Q}$ - originating from arbitrage pricing theory - to discount for the systematic risk in the cash flows. In section 5, we estimate the parameters of the risk-neutral price process using a Kalman filter. In our model, the value of the firm is represented by $V_{t}\left(x_{t}, \mathcal{P}_{t}\right)$, which is defined by the following stochastic dynamic program:

$$
\begin{aligned}
& V_{t}\left(x_{t}, \mathcal{P}_{t}\right)=\max _{z_{t}, b_{t} \in \mathcal{A}_{t}\left(x_{t}\right)} J_{t}\left(x_{t}, z_{t}, b_{t}, \mathcal{P}_{t}\right) \\
& J_{t}\left(x_{t}, z_{t}, b_{t}, \mathcal{P}_{t}\right)=\left\{\beta E_{\mathcal{P} \mid \mathcal{P}_{t}}^{\mathbb{Q}} \tilde{R}_{t}\left(b_{t}, p_{t+1}\right)\right. \\
&-\frac{\left(s_{t}+c_{t}\right)}{a}\left(z_{t}-x_{t}\right)-r\left(-x_{t}\right)^{+}-h\left(x_{t}\right)^{+} \\
&\left.+\beta E_{\mathcal{P} \mid \mathcal{P}_{t}}^{\mathbb{Q}} V_{t+1}\left(z_{t}-d_{t+1}, \mathcal{P}\right)\right\}
\end{aligned}
$$

where, $\mathcal{A}_{t}\left(x_{t}\right)=\left\{z_{t} \geq x_{t}, 0 \leq b_{t} \leq 1\right\}$, and $V_{T}\left(x_{T}, \mathcal{P}_{T}\right)$ $=\left(p_{T}-\lambda_{T}\right) x_{T}^{+}-\left(p_{T}+\lambda_{T}\right) x_{T}^{-}$.

The first term in the objective function represents the expected revenue from commodity sales. 
The second term includes the cost of procurement and processing. The third and fourth terms are penalty and holding costs, respectively. These costs are incurred at the end of the period, and are counted at the beginning of the subsequent period. The last term is the cost-to-go function under the risk-neutral measurement. According to the terminal condition, any inventory left over is sold at a discount, $p_{T}-\lambda_{T}$, and any shortages are bought at a premium, $p_{T}+\lambda_{T}$. Notice that, in the above model, all the input commodity purchased is processed and none is stored as inventory for the purpose of consumption in future periods. This is indeed an optimal processing policy because due to Assumption 2, marginal convenience yield $^{5}$ is positive, and, as a result, commodity storage with no economic use is imprudent (see, e.g., Goel and Gutierrez 2011, Williams and Wright 1991). We now characterize the optimal policies.

\subsection{Characterization of Optimal Policies}

The lemmas below first establish the concavity of the value function in the inventory level, and the joint concavity of the objective function in the decision variables.

Lemma 1. (Concavity of $\left.V_{T}\right) . V_{t}\left(x_{t}, \mathcal{P}_{t}\right)$ is concave in $x_{t}$ for every realization of $\mathcal{P}_{t}$.

Proof. It can be easily shown that $V_{T}\left(x_{T}, \mathcal{P}_{T}\right)$ is concave in $x_{T}$. Then, using concavity preservation under maximization, we establish that $V_{T-1}\left(x_{T-1}\right.$, $\left.P_{T-1}\right)$ is concave in $x_{T-1}$. The final step of the proof involves an induction argument to show that $V_{t}\left(x_{t}, P_{t}\right)$ is concave.

Lemma 2. (Joint Concavity of $\left.J_{T}\right) . J_{t}\left(x_{t}, z_{t}, b_{t}, \mathcal{P}_{t}\right)$ is strictly jointly concave in $z_{t}$ and $b_{t}$.

These results are critical in solving the dynamic program because the simultaneous solution of first-order conditions with respect to $b_{t}$ and $z_{t}$ will ensure a global maximum. We respectively define $\psi_{t}(p)$ and $\Psi_{t}(p)$ as the probability and cumulative density functions of the output commodity price at time $t$. The following theorem characterizes the optimal policy and shows that the myopic policy is optimal. All proofs appear in Appendix A, unless otherwise indicated.

THEOREM 1. The myopic policy is optimal, such that optimal $b_{t}^{*}$ and $z_{t}^{*}$ are obtained by solving the following equations: (a) if $\operatorname{Cov}\left(s_{t+1}, p_{t+1}\right)>0$

$\Psi_{t}\left(M_{t}\right)= \begin{cases}\frac{s_{t}+\beta h a-\beta f_{t}^{i}+c_{t}-\beta c_{t+1}}{\beta(r+h) a} & \text { for } t=1,2, \ldots, T-2, \\ \frac{s_{t}-\beta a f_{t}^{o}+c_{t}+\beta a \lambda_{T}}{2 \beta a \lambda_{T}} & \text { for } t=T-1,\end{cases}$

$b_{t}^{*}= \begin{cases}1-\frac{\operatorname{Cov}\left(p_{t+1}, s_{t+1}\right) / a+u_{t}\left(z_{t}^{*}, b_{t}^{*}\right)}{2 \sigma_{o}^{2}} & \text { for } t=1,2, \ldots, T-2, \\ \frac{1}{2}+\frac{\lambda_{T}}{\sigma_{0}^{2}} \int_{0}^{M_{t}}\left(f_{t}^{o}-p_{t+1}\right) \psi_{t+1}(p) d p & \text { for } t=T-1,\end{cases}$

if $b_{t}^{*}<0$ then set $b_{t}^{*}=0$ and if $b_{t}^{*}>1$ then set $b_{t}^{*}=1$, and compute (2) to obtain $z_{t}^{*}$.

(b) if $\operatorname{Cov}\left(s_{t+1}, p_{t+1}\right) \leq 0$, then $b_{t}^{*}=1$

(i) if $s_{t}-\beta f_{t}^{i} \leq \beta r a$ then $z_{t}^{*}=A_{t+1}-\gamma f_{t}^{o}-\gamma \lambda_{t+1}$ and,

(ii) if $s_{t}-\beta f_{t}^{i}>q \beta$ ra then $z_{t}^{*}=x_{t}$.

The above theorem outlines the algorithm to compute the procurement and hedging policies. The strict joint concavity result from Lemma 2 ensures that Equations (2) and (3) describe a unique solution: $z_{t}^{*}$ and $b_{t}^{*}$. The procurement policy $z_{t}^{*}$ described by Equation (2) has a newsvendor-like structure. The right-hand side of Equation (2) describes a critical ratio and the left-hand side is the probability that the output price is less than the threshold $M_{t}$, which is a function of the procurement policy $z_{t}^{*}$. The firm incurs a backlogging cost if $p_{t+1}<M_{t}$ and a holding cost if $p_{t+1}>M_{t}$. A key observation from Theorem 1 is that for $b_{t}^{*}=1$, there are no penalty and backlogging costs since perfect hedging eliminates demand uncertainty. As a result, no safety stock is required, but this policy is not necessarily optimal. Nevertheless, when the firm decides to partially hedge, $b_{t}^{*}<1$, it has to then estimate the expected backlogging and holding costs, which are a function of both the hedging policy $b_{t}^{*}$, and the procurement policy $z_{t}^{*}$. Function $u_{t}\left(z_{t}^{*}, b_{t}^{*}\right)$ calculates the expected value of the backlog plus holding costs, and it is instrumental in determining the hedging policy, as described in Equation (3). Therefore, hedging and procurement decisions are jointly determined as a function of market information on prices and firmspecific parameters. Moreover, these decisions are dynamically updated in response to changing input and output prices, as observed on the organized commodity exchanges. There are three main drivers in determining the optimal hedging policy. First, correlation between the input and output prices provides a natural hedge, reducing reliance on the financial hedge. Second, expected overstocking and understocking costs due to demand uncertainty driven by stochastic prices provide an impetus for 
hedging. Finally, the concavity of revenues in the output price is a motivation for financial hedging, as outlined in the following lemma.

\section{LeMma 3. (Jensen's IneQuality). $R_{t}\left(f_{t}^{o}\right) \geq E_{\mathcal{P} \mid \mathcal{P}_{t}}^{\mathbb{Q}}\left[R_{t}\left(p_{t+1}\right)\right]$}

According to Lemma 3, the firm's revenue function decreases in the presence of price volatility. If the firm decides to sell to the end retailer through a forward contract then it eliminates the variance in the revenue function, enhancing the expected revenue from forward sales. In other words, since the revenue function is concave in the realized price of the output commodity, the revenue decreases nonlinearly for highand low-price scenarios. Selling a forward contract to the retailer eliminates the risk of low and high price realizations, enhancing revenue. The optimal hedging strategy is a perfect hedge, $b_{t}^{*}=1$, when the correlation between the input and output price is zero, because in the absence of such correlation there is no benefit of underhedging. On the other hand, when input and output prices are correlated, the optimal hedging policy balances the expected cost of backlogging and holding with the expected benefits of the correlation between the input and output prices as well as with the benefits of hedging due to the concavity of the revenue function in the output price. The effect of the correlation between the input and output prices is summarized in the following corollary.

Corollary 1. Effect of correlation in input and output prices:

(a) If $\operatorname{Cov}\left(p_{t+1}, s_{t+1}\right)>0$ then $b_{t}^{*}<1$.

(b) If $\operatorname{Cov}\left(p_{t+1}, s_{t+1}\right)=0$ then $b_{t}^{*}=1$.

When input and output prices are positively correlated, selling in the spot market provides a natural hedge, resulting in higher profits by reducing the expected procurement cost. In our model, if the realized output price is high, this results in low demand and high inventory of ethanol (after meeting the demand). Due to a positive correlation between input and output prices, it is likely that the realization of the input price is also high. This case implies that when the price of the input is high, the firm needs to procure less since it has a high inventory of ethanol. Similarly, if the realized price of the output commodity is low, then it leads to high demand and low ethanol inventory (after meeting the demand). In this case, the firm needs to procure more (due to low inventory), but also faces a low input price. To summarize, the positive correlation between input and output prices controls the procurement cost, either through a lower input price or a lower procurement quantity. Therefore, a positive correlation between input and output prices, along with the presence of inventory across time periods, motivates the firm to underhedge and results in hedging having less value. Buying and selling forward contracts for the input commodity do not affect this result as these transactions do not affect the inherent correlation between the prices in the market.

In the finance literature, Froot et al. (1993), in a stylized single-period setting, argue that correlation between investment opportunities and cash flows of a firm results in underhedging. In a different context, our analysis yields a similar result for underhedging. Our results are established in the presence of logistical frictions, and they are driven by the correlation between the input price, output price, and demand. In addition, our model incorporates inventory and price dynamics into the firm's hedging plan, and clearly delineates the role of demand, as well as the roles of holding and shortage costs. Our findings are also related to Ho (1984) and Rolfo (1980), who show that a firm will underhedge only if there is output uncertainty in addition to price uncertainty. In contrast, our model shows that a firm will underhedge when the input and output commodity prices are positively correlated, even when there is no production uncertainty. Our results differ from Ho (1984) and Rolfo (1980) because we have an integrated view of the firm, which includes the dynamics of both input and output commodity prices in determining the optimal hedge for the output commodity. Ho (1984) and Rolfo (1980) consider only the output commodity to obtain the optimal hedge ratio. In our model, a positive correlation between input and output commodity prices provides an operational hedge that motivates the firm to underhedge the output price risk.

Theorem 2. (Value of Hedging). Suppose $\operatorname{Cov}\left(p_{t+1}\right.$, $\left.s_{t+1}\right)>0$, and let $V_{t}\left(x_{t}, \mathcal{P}_{t}\right)$ and $\bar{V}_{t}\left(x_{t}, \mathcal{P}_{t}\right)$ denote the optimal value functions under no-hedging and perfect hedging, respectively. Then, $V_{t}\left(x_{t}, \mathcal{P}_{t}\right) \leq \bar{V}_{t}\left(x_{t}, \mathcal{P}_{t}\right)$ $<V_{t}\left(x_{t}, \mathcal{P}_{t}\right)$ for every realization of $\mathcal{P}_{t}$.

The above theorem elucidates the value of hedging for a value-maximizing firm when selling to a retailer that faces demand that is negatively correlated with price. The value of hedging comes from two sources: (1) increased expected revenue from the sales of an index-based contract to the retailer by reducing price risk, and (2) better operational planning by obtaining advanced demand information through the forward sale and reducing holding and backlog costs. Notice that partial hedging dominates perfect hedging when the input and output prices are positively correlated. The covariance between the prices provides a natural hedge that renders the strategy of perfect hedging sub-optimal. In addition, hedging elicits future demand information from the downstream retailer. 
Using this information, the commodity processor makes a processing decision to reduce operational costs related to penalty and holding costs, thus creating value through advanced demand information. We also would like to note that without frictions, corporate-level risk management is irrelevant (see, e.g., Froot et al. 1993, Jin and Jorion 2006, Smith and Stulz 1985). In our case, this friction is a form of transaction cost, i.e., the logistical costs, $\lambda_{t}$, to access the exchange market (similar transaction costs have also been used in Goel and Gutierrez (2011) when justifying the use of forward contracts in commodity procurement). These logistical costs are also the driver of downward sloping demand in this study, which makes hedging relevant. Note that downward sloping demand in itself is not a market friction; it is a consequence of the logistical costs, $\lambda_{t}$. More specifically, if $\lambda_{t}=0$, then the processor can economically clear all the inventory in the exchange market and does not face a downward sloping demand. Hence, when $\lambda_{t}=0$, hedging the output price would be value-neutral since there are no other frictions in our model.

Theorem 1 also establishes the optimality of the myopic policy under three conditions: (1) absence of yield uncertainty, (2) linearly decreasing demand in the price of the output commodity, and (3) $h>h^{\prime} / a$. The absence of yield uncertainty is by construction, and once this assumption is later relaxed, we show that a myopic policy is not optimal. Considering a general demand function that decreases in price is not sufficient to show the optimality of the myopic policy. Furthermore, the condition $h>h^{\prime} / a$ ensures that it is not economical to convert input into output for storage purposes. Notice that the positive marginal convenience yield, $s_{t}+h^{\prime}-\beta f_{t}^{i} \geq 0$, ensures that it is never optimal to store input without an economic use in the current period. However, when $h<h^{\prime} / a$, it could be optimal to benefit from a negative spot-forward spread, $s_{t}-\beta f_{t}^{i}<0$, and convert input into output for storage purposes. This scenario does not lead to arbitrage, as explained in the discussion following Theorem 3, but results in the sub-optimality of the myopic policy due to a higher stocking level of output. The optimality of the myopic policy for a price taker when demand is dependent on price is a unique and significant result, particularly since it is in contrast to the results for the price setter in the literature (Federgruen and Heching 1999). The following theorem characterizes the optimal policy when $h<h^{\prime} / a$.

Theorem 3. (Storage Cost Differential). If $h<h^{\prime} / a$ then the optimal processing policy is given by a basestock level $z_{t}^{b}$ for a given $b_{t}$ and $\mathcal{P}_{t}$.

The condition $h<h^{\prime} / a$ implies that the firm has the ability to store output more efficiently than it can store input. When input prices are in contango, i.e., $s_{t}-\beta f_{t}^{i}<0$, this situation may create an opportunity for the firm to process input into output for storage purposes. In particular, this storage cost efficiency can allow the firm to trade the benefits of the contango of the input prices, $s_{t}-\beta f_{t}^{i}<0$, with the holding cost of the output to determine the optimal stocking quantity. It is important to notice that $z_{t}^{b}$ will be finite because it trades the contango of the input commodity price curve with the holding cost of the output in subsequent periods. Carrying inventory for storage purposes can result in violating the constraint $z_{t} \geq x_{t}$ rendering myopic policies sub-optimal. Following, we discuss some properties of the myopic policy described in Theorem 1.

\section{Proposition 1. (Properties of the Myopic Policy).}

(a) If $\operatorname{Cov}\left(p_{t+1}, s_{t+1}\right)>0$ then $z_{t}^{*}$ is decreasing in $\gamma$, and it is non-increasing otherwise.

(b) If $\operatorname{Cov}\left(p_{t+1}, s_{t+1}\right)>0$ then $b_{t}^{*}$ is increasing (decreasing) in $\gamma$ when $z_{t}<z_{t}^{G}\left(z_{t}>z_{t}^{G}\right)$, and it is constant otherwise, where $z_{t}^{G}=A_{t+1}-\gamma f_{t}^{o}-$ $\gamma \lambda_{t+1}$.

(c) If $\operatorname{Cov}\left(p_{t+1}, s_{t+1}\right)>0$ then $b_{t}^{*}$ is increasing in $r$ and $h$, and it is constant otherwise.

(d) If $\operatorname{Cov}\left(p_{t+1}, s_{t+1}\right)>0$ then $z_{t}^{*}$ is increasing in $r$ and decreasing in $h$, and it is constant otherwise.

As the price elasticity of demand $\gamma$ increases, the expected demand will decrease, resulting in lower amounts of the commodity being processed. If the processing quantity is below the mean demand, $z_{t}^{G}$, then as $\gamma$ increases, mean demand decreases, hence, the overage risk of the firm increases. In this case, if the covariance is positive, then $b_{t}^{*}<1$, and it is judicious for the firm to increase $b_{t}^{*}$ and reduce the variability in demand to mitigate the overage risk. On the other hand, if the processing quantity is higher than the mean demand, $z_{t}^{G}$, then as $\gamma$ increases, mean demand decreases and the risk of overage increases. To reduce the overage risk, the firm increases the variance of demand by decreasing $b_{t}^{*}$. As holding and penalty costs increase, it is optimal to hedge more to reduce the expected underage and overage costs. On the other hand, lower holding and higher penalty costs lead the firm to process more and vice versa. If the covariance is non-positive, then it is optimal to completely hedge the price risk, and the optimal solution is insensitive to changes in $r$ and $h$.

In summary, the integrated approach to commodity risk management proposed in this study has significant managerial implications. Hedging policies based only on the output commodity price risk can lead to sub-optimal results. This scenario occurs because such sub-optimal policies can disentangle the natural hedge 
provided by a positive correlation between the output and input commodity prices, resulting in lower profits. Our analysis illustrates that firms need to understand the dynamics between input and output prices across the supply chain when developing hedging policies.

So far, we have assumed that there is no yield uncertainty during the conversion process of the input commodity to the output commodity. In the following section, we incorporate yield uncertainty into commodity processing decisions to ascertain its impact on optimal processing and hedging policies.

\section{Yield Uncertainty}

In many agricultural processing environments, yield uncertainty is a challenge that production managers must deal with to ensure a regular flow of output products. The yield from processing agricultural raw materials such as corn and wheat depends upon grain quality, storage and handling, and processing parameters. We now extend our model by incorporating yield uncertainty to understand the value of financial hedging with respect to yield risk. In our model, yield uncertainty is exogenous in nature, but depends on the quantity processed. The purpose of this section is to: (1) characterize the optimal procurement policy, and (2) explore the impact of yield uncertainty on the optimal hedging policy.

According to Sobel and Babich (2012), yield uncertainty is primarily modeled in three ways: via constant variance, stochastically proportional, and binomially distributed yield. In the constant variance model, the randomness in yield is independent of the processing quantity. In the binomial model, the outcome of yield uncertainty is revealed as a sequence of binary outcomes. In our research, we follow the stochastically proportional yield model, which closely reflects the operational dynamics of ethanol processors. We define the yield of the output commodity, $\alpha\left(y_{t}\right)$, as a function of the quantity of the input commodity processed, $y_{t}$, such that $\alpha\left(y_{t}\right) \sim(a+\varepsilon) y_{t}$, where $\varepsilon \sim N(0, \sigma)$. We denote the probability distribution function of $\varepsilon$ by $\phi(\varepsilon)$. If there is no variability in the yield, then our model reduces to the model in section 3, where one bushel of corn exactly converts to $a$ gallons of ethanol. Furthermore, we assume that the risk of yield uncertainty is completely idiosyncratic and diversifiable, and it is not correlated with the market prices, such that $E_{\epsilon} E_{\mathcal{P} \mid \mathcal{P}_{t}}^{\mathbb{Q}}[\epsilon \mathcal{P}]=E_{\epsilon}[\epsilon] E_{\mathcal{P} \mid \mathcal{P}_{t}}^{\mathbb{Q}}[\mathcal{P}]$. We model commodity processing under yield uncertainty as an echelon model. We define the expected inventory position of ethanol as $\hat{z}_{t}$, such that $\hat{z}_{t}=a y_{t}+x_{t}$, and $x_{t+1}=(a+\varepsilon) y_{t}+x_{t}-d_{t+1}$. The stochastic dynamic program in Equation (1) is modified to incorporate yield uncertainty as follows:

$$
\begin{aligned}
& \hat{V}_{t}\left(x_{t}, \mathcal{P}_{t}\right)=\max _{\hat{z}_{t}, b_{t} \in \mathcal{B}_{t}\left(x_{t}\right)} \hat{J}_{t}\left(\hat{z}_{t}, b_{t}, x_{t}, \mathcal{P}_{t}\right) \\
& \hat{J}_{t}\left(\hat{z}_{t}, b_{t}, x_{t}, \mathcal{P}_{t}\right)=\left\{\beta E_{\mathcal{P} \mid \mathcal{P}_{t}}^{\mathbb{Q}} \tilde{R}_{t}\left(b_{t}, p_{t+1}\right)-\left(s_{t}+c_{t}\right) \frac{\left(\hat{z} t-x_{t}\right)}{a}\right. \\
&-r\left(-x_{t}\right)^{+}-h\left(x_{t}\right)^{+}+\beta E_{\epsilon} E_{\mathcal{P} \mid \mathcal{P}_{t}}^{\mathbb{Q}} \\
&\left.\hat{V}_{t+1}\left(x_{t+1}, \mathcal{P}\right)\right\}
\end{aligned}
$$

where, $\quad x_{t+1}=\hat{z_{t}}+\epsilon \frac{\left(\hat{z_{t}}-x_{t}\right)}{a}-d_{t+1}, \quad \mathcal{B}_{t}\left(x_{t}\right)=\left\{\hat{z}_{t} \geq\right.$ $\left.x_{t}, 0 \leq b_{t} \leq 1\right\}$, and, $\quad{ }^{b}\left(x_{T}, \mathcal{P}_{T}\right)=\left(p_{T}-\lambda_{T}\right) x_{T}^{+}-$ $\left(p_{T}+\lambda_{T}\right) x_{T}^{-}$.

The following theorem characterizes the optimal policy for procurement and hedging in the presence of yield uncertainty.

Theorem 4. (Optimal Policy Under Yield Uncertainty). For $t=1, \ldots, T-1$, let $\bar{b}_{t}$ and $\bar{z}_{t}$ be determined by simultaneously solving the following two equations:

$$
\begin{aligned}
& 2 \sigma_{o}^{2}\left(1-b_{t}\right)+\beta E_{\epsilon} E_{\mathcal{P} \mid \mathcal{P}_{t}}^{\mathbb{Q}} \frac{\partial \hat{V}_{t+1}\left(x_{t+1}, \mathcal{P}\right)}{\partial b_{t}}\left(f_{t}^{o}-p_{t+1}\right) \\
& =0 \text { and } \\
& \quad-\left(s_{t}+c_{t}\right)+\beta E_{\epsilon} E_{\mathcal{P} \mid \mathcal{P}_{t}}^{\mathbb{Q}} \frac{\partial \hat{V}_{t+1}\left(x_{t+1}, \mathcal{P}\right)}{\partial \hat{z}_{t}}=0,
\end{aligned}
$$

where $x_{t+1}=\hat{z}_{t}+\epsilon \frac{\left(\hat{z}_{t}-x_{t}\right)}{a}-d_{t+1}$. Then the firm's optimal processing policy is given by an expected base-stock level $\bar{z}_{t}^{*}$, and the hedging policy is described by $\bar{b}_{t}^{*}$, such that (i) if $\bar{b}_{t} \in[0,1]$ then $\bar{z}_{t}^{*}=\bar{z}_{t}$ and $\bar{b}_{t}^{*}=\bar{b}_{t}$ and (ii) if $\bar{b}_{t} \notin[0,1]$ then $\bar{b}_{t}^{*}=\max \left\{\min \left\{\bar{b}_{t}, 1\right\}, 0\right\}$, and $\bar{z}_{t}^{*}$ is obtained by solving Equation (6).

Incorporating yield uncertainty into our analysis results in the sub-optimality of the myopic policy as it may violate the constraint $\hat{z}_{t} \geq x_{t}$. Our approach in defining an expected base-stock level is similar to Sobel and Babich (2012), who define echelon-like basestock levels. Nevertheless, the main distinction between our model and theirs is that we model stochastically proportional yield (while they model yield with a constant variance) and our model incorporates stochasticity in prices. In our case, any attempt to model yield with a constant variance will not result in the optimality of the myopic policy, as in Sobel and Babich (2012) does, for two key reasons. First, Assumption 2 in Sobel and Babich (2012) is not applicable because demand in our model is price dependent, and prices are mean reverting, such that price shocks are not independent and identically distributed. Second, Property 1 in Sobel and Babich (2012) cannot be applied to our model because price is a log-normal random variable, and the futures price is not linear in the current state. Therefore, future expected inventory cannot be written as a linear combination of past states of inventory and price. Notice that our modeling approach for yield will be 
intractable under a higher number of echelons due to the "curse of dimensionality." Nevertheless, we are able to characterize an optimal policy in a twoechelon structure because in our model there is no incentive to carry inventory at the upper echelon due to marginal convenience yield, which reduces the problem to a single echelon.

The complication in solving Equations (5) and (6) is that the transition probabilities from state $\mathcal{P}_{t}$ to $\mathcal{P}_{t+1}$ are not easy to compute, particularly when the price process is at least two-dimensional with one dimension each for input and output commodity prices. In section 5 , we discretize the price process through a binomial lattice to calculate Equations (5) and (6) in order to compute $\bar{z}_{t}^{*}$ and $\bar{b}_{t}^{*}$. Nevertheless, as the time horizon for decision making increases, the size of the binomial lattice increases. Due to the curse of dimensionality it becomes computationally challenging to numerically calculate the dynamic program. As a result, we aspire to obtain myopic policies as an approximation of the optimal policy. The following theorem develops the myopic policy for the model defined in Equation (4).

Theorem 5. (Myopic Policy Under Yield Uncertainty). Let $\bar{b}_{t}$ and $\bar{z}_{t}$ be determined by simultaneously solving the following two equations:

(a) for $t=1,2, \ldots, T-2$

$$
\begin{aligned}
\Gamma\left(\hat{M}_{t}\right) & =\frac{s_{t}+a \beta h-\beta f_{t}^{i}+c_{t}-\beta c_{t+1}}{a \beta(r+h)} \\
2 \sigma_{o}^{2}\left(1-b_{t}\right) & =\operatorname{Cov}\left(s_{t+1}, p_{t+1}\right) / a+g\left(\hat{z}_{t}, b_{t}\right),
\end{aligned}
$$

(b) for $t=T-1$

$$
\begin{gathered}
\Gamma\left(\hat{M}_{t}\right)=\frac{s_{t}+c_{t}-a \beta f_{t}^{0}+a \beta \lambda_{T}}{2 \beta a \lambda_{T}} \\
b_{t}=\frac{1}{2}+\frac{\lambda_{T}}{\sigma_{0}^{2}} \iint_{0}^{\hat{M}_{t}}\left(f_{t}^{o}-p_{t+1}\right) \psi_{t+1}(p) d p \phi(\epsilon) d \epsilon,
\end{gathered}
$$

where $g\left(\hat{z}_{t}, b_{t}\right)=\int\left\{-r \int_{0}^{\hat{M}_{t}}\left(f_{t}^{o}-p_{t+1}\right) \psi_{t+1}(p) d p+h \int_{\hat{M}_{t}}^{\infty}\right.$ $\left.\left(f_{t}^{o},-p_{t+1}\right) \psi_{t+1}(p) d p\right\} \phi(\epsilon) d \epsilon, \Gamma\left(\hat{M}_{t}\right)=\int\left(1+\frac{\epsilon}{a}\right) \Psi_{t+1}\left(\hat{M}_{t}\right)$ $\phi(\epsilon) d \epsilon$, and $\hat{M}_{t}=\frac{A_{t+1}-\gamma b_{t} f_{t}^{o}-\gamma \lambda_{t+1}-\hat{z}_{t}-\epsilon\left(\hat{z}_{t}-x_{t}\right) / a}{\gamma\left(1-b_{t}\right)}$. Then, myopic procurement and hedging policies $\hat{z}_{t}^{*}$ and $\hat{b}_{t}^{*}$, respectively, can be obtained, such that (i) if $\bar{b}_{t} \in[0,1]$ then $\hat{z}_{t}^{*}=\bar{z}_{t}$ and $\hat{b}_{t}^{*}=\bar{b}_{t}$ and (ii) if $\bar{b}_{t} \notin[0,1]$ then $\hat{b}_{t}^{*}=\max \left\{\min \left\{\bar{b}_{t}, 1\right\}, 0\right\}$, and $\hat{z}_{t}^{*}$ is obtained by solving the above equations.

Our objective is to explore the conditions under which the myopic policy performs close to the optimal policy. In this regard, we expect to experience two effects, namely the propagation effect and the look-ahead effect. Under the optimal policy, the firm processes a higher quantity (compared to the myopic policy) to carry inventory in earlier periods as a hedge against poor yield outcomes in later periods. We call this effect the propagation effect, and we expect it to be amplified under a low holding cost. In the presence of the propagation effect, we expect the myopic policy to perform poorly. In addition, as the holding cost increases, the cost of mismatch between the myopic and optimal policies increases, decreasing the performance of the myopic policy. This situation is called the look-ahead effect because it only occurs when the constraint $\hat{z}_{t} \geq x_{t}$ is violated, as myopic policies are not forward looking. As a result, we expect myopic policies to perform better under a moderate holding cost. The numerical analysis ${ }^{6}$ presented in Table 1 corroborates our intuition. In addition, the myopic policy performs poorly as yield uncertainty increases.

\section{Numerical Analysis}

In section 5.1, we describe the stochastic price processes used to jointly model the input and output commodity prices, and describe the method to estimate the price process parameters for corn and ethanol using the futures price data from the CME. Then, in section 5.2 we discuss the discretization of the price process and the algorithm to compute the dynamic program. In section 5.3, we discuss the managerial insights generated through the sensitivity analysis of the price process parameters and firm characteristics.

\subsection{Stochastic Price Process}

We model the input and output commodity prices as a mean-reverting stochastic process. In particular, we model the logarithm of the price of a commodity as an Ornstein-Uhlenbeck process, defined as $d \chi_{t}^{i}=$ $\kappa^{i}\left(\alpha^{i}-\chi_{t}^{i}-\lambda^{i}\right) d t+\sigma^{i} d Z^{i}, d \chi_{t}^{o}=\kappa^{o}\left(\alpha^{0}-\chi_{t}^{o}-\lambda^{o}\right) d t$ $+\sigma^{o} d Z^{o}$, and $d Z^{i} \cdot d Z^{o}=\rho d t$. Superscripts $i$ and $o$ represent input and output commodities, respectively. $\chi$ is the $\log$ of the price, $\kappa$ represents the rate of mean reversion, $\alpha$ is the long-run average price, $\lambda$ is the risk premium per unit of mean reversion, $\sigma$ denotes the volatility in the commodity price, $\rho$ represents the instantaneous correlation between the two stochastic processes, and $d Z$ is the increment of a Brownian

Table 1 Percentage Difference in Value Function between Myopic and Optimal Policies

\begin{tabular}{lllllr}
\hline & \multicolumn{5}{c}{ Coefficient of variation in yield } \\
\cline { 2 - 6 } Holding cost & 0.04 & 0.06 & 0.08 & 0.10 & \multicolumn{1}{c}{0.12} \\
\hline 0.01 & 0.99 & 3.19 & 7.62 & 10.70 & 14.00 \\
0.02 & 0.84 & 0.82 & 3.13 & 5.64 & 11.00 \\
0.04 & 0.82 & 0.62 & 2.68 & 3.50 & 4.50 \\
0.10 & 0.84 & 0.58 & 2.68 & 3.37 & 4.20 \\
0.20 & 0.88 & 0.59 & 3.27 & 4.57 & 7.00 \\
0.30 & 0.93 & 0.61 & 4.28 & 7.15 & 11.00 \\
\hline
\end{tabular}


Motion associated with the stochastic process. Under the risk-neutral measure, the futures price at time $t$ for a contract that expires at time $\tau$ is defined as $f_{t, \tau}^{i}=\exp \left[\chi_{t}^{i} \mathrm{e}^{-\kappa^{i}(\tau-t)}+\left(\alpha^{i}-\lambda^{i}\right)\left(1-\mathrm{e}^{-\kappa^{i}(\tau-t)}\right)+\frac{\left(\sigma^{i}\right)^{2}}{4 \kappa^{i}}\right.$ $\left.\left(1-\mathrm{e}^{-2 \kappa^{i}(\tau-t)}\right)\right]$ for the input commodity, and $f_{t, \tau}^{o}=\exp \left[\chi_{t}^{o} \mathrm{e}^{-\kappa^{o}(\tau-t)}+\left(\alpha^{o}-\lambda^{o}\right)\left(1-\mathrm{e}^{-\kappa^{o}(\tau-t)}\right)+\frac{\left(\sigma^{o}\right)^{2}}{4 \kappa^{o}}\right.$ $\left.\left(1-\mathrm{e}^{-2 \kappa^{\circ}(\tau-t)}\right)\right]$ for the output commodity.

We estimate the parameters of the price process by applying a Kalman filter technique on the futures price data for corn and ethanol from the CME between 4/1/2005 and 12/31/2011. According to the corn futures price data, the average price for corn is 300 cents/bushel. Corn futures contracts typically mature in the months of March, May, July, September, and December, and there are about 15 such contracts traded at a time for various maturities. The average price for ethanol is 120 cents/gallon. Ethanol futures have been trading on the CME since early 2005, and the contracts mature every month. Table 2 illustrates the parameters of the joint stochastic price process for the two commodities (see Schwartz and Smith 2000 for details). We observe moderate levels of mean reversion for both commodities. In general, the meanreversion factor is difficult to estimate, but we observe from the low values of the standard deviation that the coefficient of mean reversion is significant. The volatility of the two commodities is around $30 \%$, with a strong correlation in prices. The parameters in Table 2 are used in the numerical section to compute the futures prices and conduct a sensitivity analysis on the optimal procurement and hedging policies.

\subsection{Optimization Algorithm}

To compute the optimal procurement and hedging policies, we discretize the price process on a binomial lattice as a function of the input and output price variables. We then solve the dynamic program using Theorem 4 to obtain the optimal policies. We discretize the stochastic price process as a recombinant lattice, as in Kamrad and Ritchken (1991). The state space of price $\mathcal{P}_{t}$ is a function of $\chi_{t}^{i}$ and $\chi_{t}^{o}$. We define $\hat{\mathcal{P}}_{t}$ as the lattice nodes, such that $\hat{\mathcal{P}}_{t} \in \mathcal{P}_{t}$, where $\hat{\mathcal{P}}_{t} \equiv\left(\hat{\chi}_{t}^{i}, \hat{\chi}_{t}^{o}\right)$. The jump size on the lattice corresponding to the input commodity is denoted by $\Delta^{i}$, and it is given by $\Delta^{i}=\sigma^{i} \sqrt{\Delta t}$, where $\Delta t$ is the time interval between successive jumps. Similarly, the jump size on the lattice corresponding to the output commodity is denoted by $\Delta^{o}$, and it is given by $\Delta^{o}=\sigma^{o} \sqrt{\Delta t}$. The lattice starts at time $t=0$ from node $\hat{\mathcal{P}}_{0} \equiv\left(\hat{\chi}_{0}^{i}, \hat{\chi}_{0}^{o}\right)$ and then transitions to four possible nodes. From every state node $\hat{\mathcal{P}}_{t} \in \mathcal{P}_{t}$, we consider the possibility of four transitions as $\hat{\mathcal{P}}_{t}^{ \pm \pm} \epsilon \mathcal{P}_{t+1}$, defined as $\hat{\mathcal{P}}_{t}^{ \pm \pm}=\left\{\hat{\chi}_{t}^{i} \pm \Delta^{i}, \hat{\chi}_{t}^{o} \pm \Delta^{o}\right\}$, depending upon the up or down jump for the combination of input and output prices.

The transition probabilities of mean-reverting processes, such as an Ornstein-Uhlenbeck process, are known to be state dependent. We denote $P_{\hat{\mathcal{P}}_{t}}^{ \pm \pm}$as the transition probability under the risk-neutral measure from node $\hat{\mathcal{P}}_{t}$ to $\hat{\mathcal{P}}_{t}^{ \pm \pm}$. As an example, $P_{\hat{\mathcal{P}}_{t}}^{+-}$represents the transition probability from node $\hat{\mathcal{P}}_{t} \in \mathcal{P}_{t}$ to an up node for the input commodity and a down node for the output commodity. These four probabilities at each node are obtained by requiring them to sum up to 1 , and equating the risk-neutral conditional expectations, variance and covariance of the discretized process to those of the original process, as described in Kamrad and Ritchken (1991) and Hahn and Dyer (2008). Nevertheless, as the transition probabilities are state dependent, they may be required to censor. In this regard, we follow the two-step approach of Hahn and Dyer (2008) to develop the conditional transition probabilities. The jump probabilities from the state variable $\hat{\chi}_{t}^{i}$ are defined as $P\left\{\hat{\chi}_{t}^{i}+\Delta^{i}\right\}=P_{\hat{\chi}_{t}^{i}}^{+}$and $P\left\{\hat{\chi}_{t}^{i}-\Delta^{i}\right\}=P_{\hat{\chi}_{t}^{i}}^{-}$. Using Bayes' rule, $P\left\{\hat{\chi}_{t}^{0}+\right.$ $\left.\Delta^{o} \mid \hat{\chi}_{t}^{i}+\Delta^{i}\right\}=P_{\hat{\mathcal{P}}_{t}}^{++} / P_{\hat{\chi}_{t}^{i}}^{+} \quad P\left\{\hat{\chi}_{t}^{o}+\Delta^{o} \mid \hat{\chi}_{t}^{i}-\Delta^{i}\right\}=P_{\hat{\mathcal{P}}_{t}}^{-+} /$ $P_{\hat{\chi}_{t}^{i}}^{-} \quad P\left\{\hat{\chi}_{t}^{o}-\Delta^{o} \mid \hat{\chi}_{t}^{i}+\Delta^{i}\right\}=P_{\hat{\mathcal{P}}_{t}}^{+-} / P_{\hat{\chi}_{t}^{\prime \prime}}^{+} \quad$ and $\quad P\left\{\hat{\chi}_{t}^{o}-\right.$ $\left.\Delta^{o} \mid \hat{\chi}_{t}^{i}-\Delta^{i}\right\}=P_{\hat{\mathcal{P}}_{t}}^{--} / P_{\hat{\chi}_{t}^{i}}^{-}$. The conditional transition probabilities are given as:

$$
\begin{aligned}
P\left\{\hat{\chi}_{t}^{i}+\Delta^{i}\right\} & =\frac{1}{2}\left[1+\frac{\kappa^{i}\left(\alpha^{i}-\lambda^{i}-\hat{\chi}_{t}^{i}\right)}{\sigma^{i}} \sqrt{\Delta t}\right] \\
P\left\{\hat{\chi}_{t}^{i}-\Delta^{i}\right\} & =\frac{1}{2}\left[1-\frac{\kappa^{i}\left(\alpha^{i}-\lambda^{i}-\hat{\chi}_{t}^{i}\right)}{\sigma^{i}} \sqrt{\Delta t}\right] \\
P\left\{\hat{\chi}_{t}^{o}+\Delta^{o} \mid \hat{\chi}_{t}^{i}+\Delta^{i}\right\} & =\frac{1}{2}\left[1+\frac{\left(\kappa^{o}\left(\alpha^{o}-\lambda^{o}-\hat{\chi}_{t}^{o}\right) \sqrt{\Delta t}+\rho \sigma^{o}\right) \sigma^{i}}{\sigma^{o}\left(\sigma^{i}+\kappa^{i}\left(\alpha^{i}-\lambda^{i}-\hat{\chi}_{t}^{i}\right) \sqrt{\Delta t}\right)}\right] \\
P\left\{\hat{\chi}_{t}^{o}+\Delta^{o} \mid \hat{\chi}_{t}^{i}-\Delta^{i}\right\} & =\frac{1}{2}\left[1+\frac{\left(\kappa^{o}\left(\alpha^{o}-\lambda^{o}-\hat{\chi}_{t}^{o}\right) \sqrt{\Delta t}-\rho \sigma^{o}\right) \sigma^{i}}{\sigma^{o}\left(\sigma^{i}-\kappa^{i}\left(\alpha^{i}-\lambda^{i}-\hat{\chi}_{t}^{i}\right) \sqrt{\Delta t}\right)}\right] \\
P\left\{\hat{\chi}_{t}^{o}-\Delta^{o} \mid \hat{\chi}_{t}^{i}+\Delta^{i}\right\} & =\frac{1}{2}\left[1-\frac{\left(\kappa^{o}\left(\alpha^{o}-\lambda^{o}-\hat{\chi}_{t}^{o}\right) \sqrt{\Delta t}+\rho \sigma^{o}\right) \sigma^{i}}{\sigma^{o}\left(\sigma^{i}+\kappa^{i}\left(\alpha^{i}-\lambda^{i}-\hat{\chi}_{t}^{i}\right) \sqrt{\Delta t}\right)}\right] \\
P\left\{\hat{\chi}_{t}^{o}-\Delta^{o} \mid \hat{\chi}_{t}^{i}-\Delta^{i}\right\} & =\frac{1}{2}\left[1-\frac{\left(\kappa^{0}\left(\alpha^{o}-\lambda^{o}-\hat{\chi}_{t}^{o}\right) \sqrt{\Delta t}-\rho \sigma^{o}\right) \sigma^{i}}{\sigma^{o}\left(\sigma^{i}-\kappa^{i}\left(\alpha^{i}-\lambda^{i}-\hat{\chi}_{t}^{i}\right) \sqrt{\Delta t}\right)}\right] .
\end{aligned}
$$

Table 2 Estimated Stochastic Price Process Parameters

\begin{tabular}{lccccccrr}
\hline Symbol & $\alpha^{i}$ & $\kappa^{i}$ & $\lambda^{i}$ & $\sigma^{i}$ & $\alpha^{0}$ & $\kappa^{o}$ & $\lambda^{o}$ & $\sigma^{o}$ \\
\hline Mean & 6.505 & 0.170 & 0.579 & 0.323 & 5.532 & 0.142 & 0.552 & 0.275 \\
SE & 0.797 & 0.007 & 0.799 & 0.019 & 0.833 & 0.010 & 0.842 & 0.013 \\
\hline
\end{tabular}


We discretize the price process on two factors, such that we compute $t^{2}$ nodes at time $t$. For our numerical analysis, we also truncate the price distribution from the above by the monopoly price, to ensure that the firm remains a price taker. The outline of the algorithm that calculates (4) is as follows:

\section{Step 1: Initialization}

(a) Set up the lattice to discretize the price process, and calculate the transition probabilities from $\hat{\mathcal{P}}_{t} \in \mathcal{P}_{t}$ to the corresponding nodes in $\hat{P}_{t+1}$.

(b) Censor the transition probabilities based on Equation (7).

(c) At each node, calculate $f_{t, t+1}^{o}=\exp \left[\hat{\chi}_{t}^{0} \mathrm{e}^{-\kappa^{0}}\right.$ $\left.+\left(\alpha^{o}-\lambda^{o}\right)\left(1-\mathrm{e}^{-\kappa^{o}}\right)+\frac{\left(\sigma^{o}\right)^{2}}{4 \kappa^{o}}\left(1-\mathrm{e}^{-2 \kappa^{o}}\right)\right]$.

\section{Step 2: Recursive Calculation}

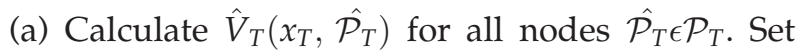
$t=T-1$.

(b) Calculate $E_{\hat{\mathcal{P}} \mid \hat{\mathcal{P}}_{t}}^{\mathbb{Q}} \hat{V}_{t+1}\left(x_{t+1}, \hat{\mathcal{P}}\right)$ to obtain $\bar{z}_{t}^{*}$ and $\bar{b}_{t}^{*}$ from Theorem 4 for all nodes $\hat{\mathcal{P}}_{t} \in \mathcal{P}_{t}$. Then calculate $\hat{V}_{t}\left(x_{t}, \hat{\mathcal{P}}_{t}\right)$.

(c) Set $t=t-1$. If $t>0$ then go to step 2b: otherwise stop.

A similar algorithm can be used to compute the optimal policies and the value function for the case without yield uncertainty using Theorem 1 . We next develop managerial insights based on the numerical analysis.

\subsection{Managerial Insights}

In this section, to gain further managerial insights, we conduct a sensitivity analysis for the parameters, such as the volatility of input and output commodity prices, the correlation between the prices, and the holding cost. We consider a planning horizon of $T=10$ weeks. ${ }^{7}$ We change one parameter at a time while keeping the other parameters at their base-case values, as detailed in Table 2 . We first develop managerial insights for the deterministic yield case, and then examine the impact of yield uncertainty. We compute the value function of selling through the spot market, defined as $V($ spot), using Equation (1) when $b_{t}=0$. Similarly, we compute the value of the optimal policy, defined as V(optimal), using Equation (1). Then we denote their percentage difference on graphs, such that $\%$ Gains $=\frac{V(\text { optimal })-V(\text { spot })}{V(\text { spot })} \times 100$.

Effect of output and input commodity price volatility: Hedging creates more value as the volatility of the output commodity price increases, as shown in Figure 1a. This result is driven by the concavity of the revenues due to the negative correlation between the demand and output price, which creates a bigger incentive to hedge as the output price becomes more volatile. On the other hand, the effect of input price volatility is driven by a different mechanism. As the input price becomes more volatile, the effect of selfhedging due to correlated prices becomes more pronounced. In particular, higher input price volatility creates more opportunities to reduce the procurement cost when the firm is not hedging due to correlation between the prices. This situation increases the benefits of underhedging leading to lower motivation to hedge. Hence, as the volatility of the input price increases, the value of hedging decreases, as shown in Figure 1c. This is a unique result of this study. In addition, this effect gets further amplified with an increase in demand elasticity $\gamma$.

Effect of price correlation and holding cost: When input and output prices are positively correlated, selling in the spot market provides a natural hedge, resulting in higher profits by reducing the expected procurement cost. In our model, if the realized output price is high, this results in low demand and high inventory of ethanol. Due to a positive correlation between input and output prices, it is likely that the realization of the input price is also high. This case implies that when the price of the input is high, the firm needs to procure less since it has a high inventory of ethanol. Similarly, if the realized price of the output commodity is low, then it leads to high demand and low ethanol inventory. In this case, the firm needs to procure more, but also faces a low input price. To summarize, the positive correlation between input and output prices controls the procurement cost, either through a lower input price or a lower procurement quantity. Therefore, as the correlation increases, the firm gets more motivated to sell in the spot market resulting in a lower value of hedging, as shown in Figure 1b. On the other hand, the value of hedging increases with higher holding costs, as illustrated in Figure 1d. A higher degree of hedging allows better operational efficiency by eliciting advanced demand information, resulting in less mismatch in demand and supply. An increase in the holding cost leads to a higher cost for a supply and demand mismatch, resulting in a higher value from hedging. Similar results can be shown for the penalty cost.

Effect of yield uncertainty: The value of the firm decreases with an increase in yield uncertainty. This result is consistent with the economics and finance literature. Nevertheless, the percentage benefit of hedging is non-monotonic in yield uncertainty, as evident from Figure 2. In Figure 2a, the base case refers to the base-case volatility of corn, and the other cases refer to the $80 \%$ and $120 \%$ volatility of corn with respect to the base case. Similarly, in Figure $2 b$, the base case refers to the base-case volatility of ethanol, and the other cases refer to the $90 \%$ and $110 \%$ volatility of ethanol in the base case. As yield uncertainty 
Figure 1 Effect of Hedging on Firm Value [Color figure can be viewed at wileyonlinelibrary.com]

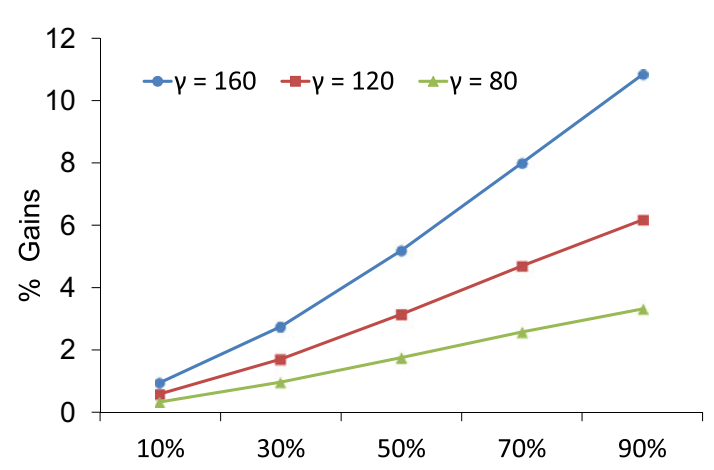

(a) Ethanol Volatility (\% Annual Volatility)

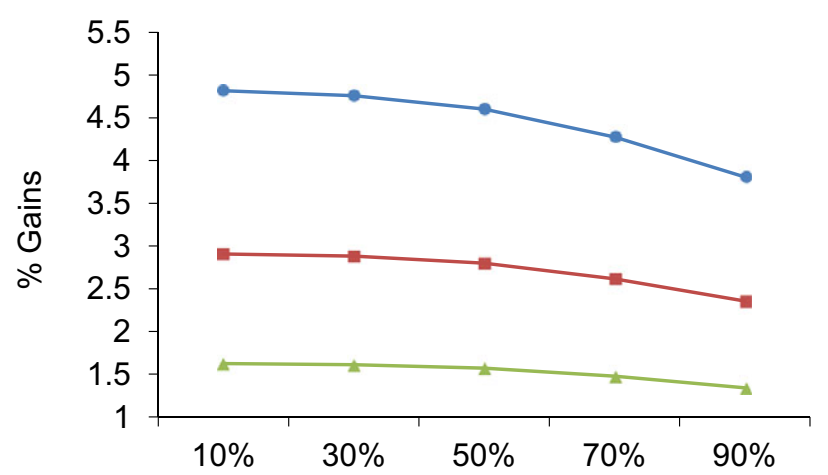

(c) Corn Volatility (\% Annual Volatility)

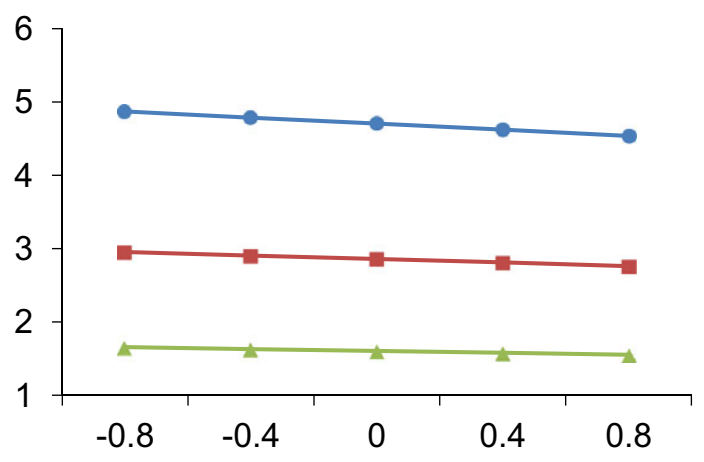

(b) Correlation in Input-Output Price

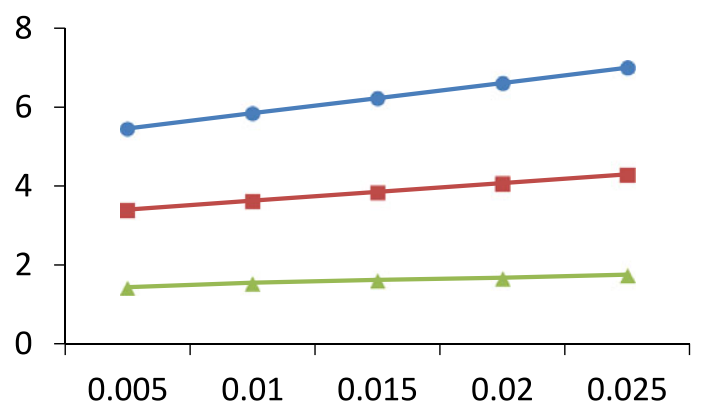

(d) Holding Cost (Cents/Week)

Figure 2 Effect of Yield Uncertainty on the \% Benefit of Hedging [Color figure can be viewed at wileyonlinelibrary.com]

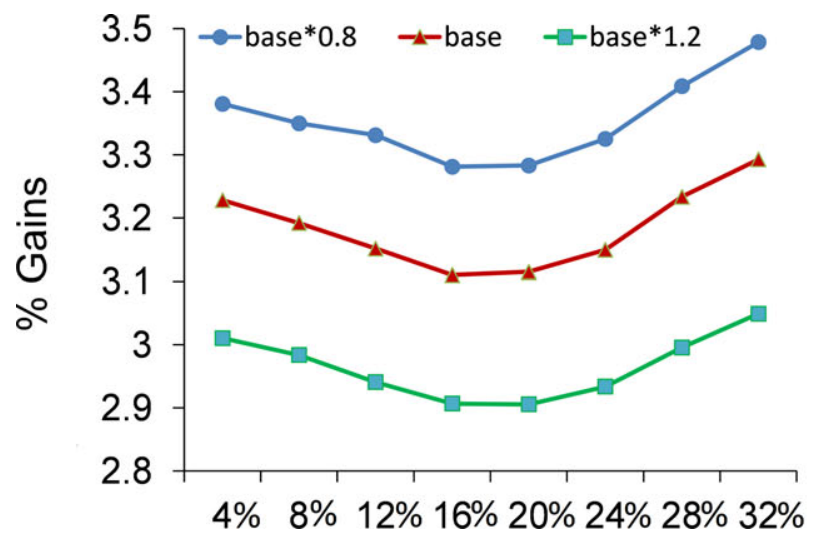

(a) Yield Uncertainty and Corn Volatility

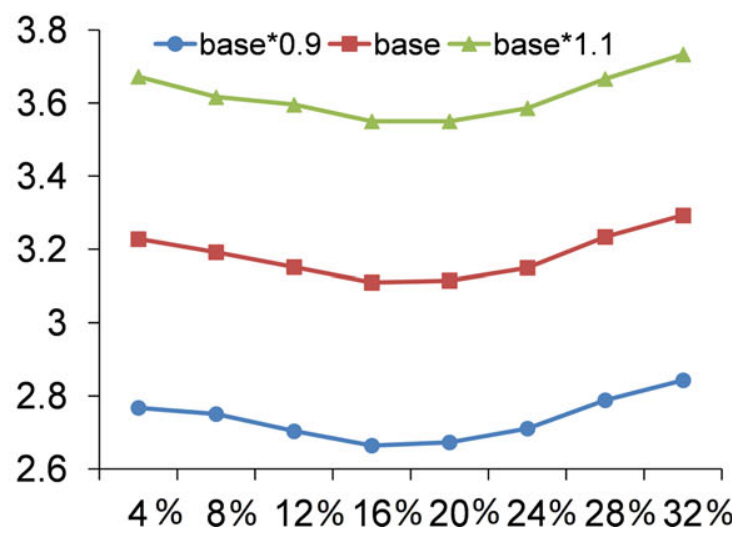

(b) Yield Uncertainty and Ethanol Volatility increases, it increases the risk exposure of the firm, but only market risk can be hedged through the contract. Therefore, the percentage of risk that can be hedged decreases with an increase in yield uncertainty, decreasing the value of hedging. However, as yield uncertainty becomes very high, the percentage benefit of hedging increases as the value of firm, which relies purely on spot procurement, decreases sharply. As a result, the percentage benefit of hedging is non-monotonic in yield uncertainty.

\section{Concluding Remarks}

We consider the operations of a commodity processor that is a price taker in the commodity markets. In general, commodity processors operate with thin 
profit margins, making it imperative to implement optimal procurement, processing, and hedging policies. We formulate a multi-period model where the processor procures an input commodity in the spot market to process and sell it to the downstream retailer. The commodity processor may sell the output through a spot, forward or an index-based contract. In this study, we jointly optimize procurement policies for the input commodity, and financial hedging policies for the output commodity when demand is negatively correlated with output price. We also assume that the input and output commodity prices are correlated and follow a joint stochastic process that offers no risk-free arbitrage opportunities. In summary, we develop an integrated risk management model for the commodity processor that accounts for correlation between demand and the output commodity price, and also captures the correlation between input and output prices.

Under this integrated framework, we show that in general, neither selling exclusively in spot nor forward markets is optimal, but selling through an index price, which is a combination of spot and forward prices, is optimal. This leads to an optimal hedge ratio of less than 1 , which is in contrast to the classic economics literature that considers optimizing only the output end of the supply chain and concludes that the optimal hedge ratio is one in the absence of yield uncertainty (Ho 1984, Rolfo 1980). Our research concludes that the correlation between input and output prices provides a natural hedge, resulting in a decrease in reliance on financial hedging. One of the key managerial insights of our research is that hedging is most beneficial when output price volatility is high and input price volatility is low.

Financial theory explains the value of hedging through capital market imperfections, such as bankruptcy costs, taxation, agency problems, and inefficient pricing of derivatives. In our study, we consider a form of friction, that is, logistical costs, $\lambda_{t}$, to access the exchange market. This logistical cost is also the driver of the downward sloping demand in the study. In particular, there are two distinct reasons why hedging creates value in our value-maximization framework: (1) Logistical costs result in the nonlinearity of the profit function in the output price, leading to the optimality of hedging. (2) Hedging elicits demand information from the downstream retailer to allow efficient operational planning, eliminating wasteful inventory due to a mismatch in demand and supply.

We identify three conditions under which a myopic policy is optimal: (1) absence of yield uncertainty, (2) linearly decreasing demand in the price of the output commodity, and (3) more expensive storage of the output commodity than the input commodity. These results are significant because they are contrary to the existing literature on price-setter firms, where orderup-to policies have been shown to be optimal. For a given hedging policy, the optimal input commodity procurement policy has a newsvendor-like structure as a function of the spot and futures prices of the input commodity. Our research also elucidates the role of the term structure of futures prices on the optimal procurement policy.

Agricultural commodity processors also deal with yield uncertainty in pursuit of matching supply with demand. In the presence of such uncertainty, however, a myopic policy is not optimal. We model yield uncertainty as stochastically proportional to the processing quantity, and show that an expected basestock policy is optimal. As the time horizon of decision making increases, the state space of the joint price process on the lattice increases exponentially, rendering it impossible to compute the optimal policy due to the curse of dimensionality. In this context, we develop myopic policies and conclude that they perform reasonably well for moderate values of holding cost. However, their performance deteriorates as the yield becomes more uncertain. This is the first paper in the operations literature that studies hedging under yield uncertainty. We find that yield uncertainty has a U-shaped effect on the benefits of hedging.

This research contributes to the growing literature at the interface of operations and finance. Our analysis concludes that the correlation coefficient between the input and output prices is key in determining the optimal hedging policy. In this study, we assume a static correlation coefficient between prices. Modeling a stochastic correlation coefficient as an additional factor in the price process would generate additional insights. Furthermore, we show that index-based contracts create value for a firm, and it could be further investigated how other financial contracts, such as swaps, options, or swing options, could be used by a firm to create value. The role of capacity constraints on optimal hedging policies could also be explored further. We believe that the managerial insights developed from our analysis will be useful for procurement and sales managers in a commodity supply chain.

\section{Appendix A. Proofs}

\section{A.1. Proof of Lemma 2}

PROOF. The proof is by backward induction. For this purpose, we first need to show that $J_{t}\left(x_{t}, z_{t}, b_{t}, \mathcal{P}_{t}\right)$ is jointly concave in $z_{t}$ and $b_{t}$ for 
$t=T-1$, i.e., for the last decision period. Recall that $J_{T-1}\left(x_{T-1}, z_{T-1}, b_{T-1}, \mathcal{P}_{T-1}\right)$ is defined as:

$$
\begin{aligned}
& J_{T-1}\left(x_{T-1}, z_{T-1}, b_{T-1}, \mathcal{P}_{T-1}\right)=\beta E_{\mathcal{P} \mid \mathcal{P}_{T-1}}^{\mathbb{Q}} \tilde{R}_{T-1}\left(b_{T-1}, p_{T}\right) \\
& -\frac{\left(s_{T-1}+c_{T-1}\right)}{a}\left(z_{T-1}-x_{T-1}\right)-r\left(-x_{T-1}\right)^{+} \\
& -h\left(x_{T-1}\right)^{+}+\beta E_{\mathcal{P} \mid \mathcal{P}_{T-1}}^{\mathbb{Q}} V_{T}\left(z_{T-1}-d_{T}, \mathcal{P}\right),
\end{aligned}
$$

where $\quad V_{T}\left(x_{T}, \mathcal{P}_{T}\right)=\left(p_{T}-\lambda_{T}\right) x_{T}^{+}-\left(p_{T}+\lambda_{T}\right) x_{T}^{-}$ and $x_{T}=z_{T-1}-d_{T}$.

The first-order partial derivative of $J_{T-1}\left(x_{T-1}\right.$, $\left.z_{T-1}, b_{T-1}, \mathcal{P}_{T-1}\right)$ with respect to $z_{T-1}$ is shown below (for brevity, we will not index $\psi$ and $\Psi$, and omit the arguments of the function $\left.J_{T-1}\left(x_{T-1}, z_{T-1}, b_{T-1}, \mathcal{P}_{T-1}\right)\right)$.

$$
\begin{aligned}
\frac{\partial J_{T-1}}{\partial z_{T-1}}= & -\frac{s_{T-1}+c_{T-1}}{a}+\beta\left(f_{T}^{o}+\lambda_{T}\right) \int_{0}^{M_{T-1}} \psi(p) d p \\
& +\beta\left(f_{T}^{o}-\lambda_{T}\right) \int_{M_{T-1}}^{\infty} \psi(p) d p,
\end{aligned}
$$

where $M_{T-1}=\frac{A_{T}-\gamma b_{T-1} f_{T-1}^{0}-\gamma \lambda_{T}-z_{T-1}}{\gamma\left(1-b_{T-1}\right)}$. Following, the second-order partial derivative of $J_{T-1}$ with respect to $z_{T-1}$ is given by:

$$
\frac{\partial^{2} J_{T-1}}{\partial z_{T-1}^{2}}=2 \beta \lambda_{T} \frac{\partial M_{T-1}}{\partial z_{T-1}} \psi\left(M_{T-1}\right)
$$

Observing $\frac{\partial M_{T-1}}{\partial z_{T-1}}=-\frac{1}{\gamma\left(1-b_{T-1}\right)}<0$, we conclude that $\frac{\partial^{2} J_{T-1}}{\partial z_{T-1}^{2}}=-2 \beta \lambda_{T} \frac{1}{\gamma\left(1-b_{T-1}\right)} \psi\left(M_{T-1}\right)<0$. Similarly, the first-order partial derivative of $J_{T-1}$ with respect to $b_{T-1}$ is shown below for $0 \leq b_{T-1} \leq 1$.

$$
\begin{aligned}
\frac{\partial J_{T-1}}{\partial b_{T-1}}= & 2 \gamma\left(1-b_{T-1}\right) \sigma_{o}^{2} \\
& +\gamma \beta \int_{0}^{M_{T-1}}\left(f_{T-1}^{o}-p_{T}\right)\left(p_{T}+\lambda_{T}\right) \psi(p) d p \\
& +\gamma \beta \int_{M_{T-1}}^{\infty}\left(f_{T-1}^{o}-p_{T}\right)\left(p_{T}-\lambda_{T}\right) \psi(p) d p .
\end{aligned}
$$

Following, the second-order partial derivative of $J_{T-1}$ with respect to $b_{T-1}$ is given by:

$$
\frac{\partial^{2} J_{T-1}}{\partial b_{T-1}^{2}}=-2 \gamma \sigma_{o}^{2}+2 \gamma \beta \lambda_{T} \frac{\partial M_{T-1}}{\partial b_{T-1}}\left(f_{T-1}^{o}-M_{T-1}\right) \psi\left(M_{T-1}\right) .
$$

Then, substituting for

$$
M_{T-1}=\frac{A_{T}-\gamma b_{T-1} f_{T-1}^{0}-\gamma \lambda_{T}-z_{T-1}}{\gamma\left(1-b_{T-1}\right)}
$$

and

$$
\frac{\partial M_{T-1}}{\partial b_{T-1}}=-\frac{\gamma f_{T-1}^{o}-A_{T}+\gamma \lambda_{T}+z_{T-1}}{\gamma\left(1-b_{T-1}\right)^{2}},
$$

we obtain:

$$
\begin{aligned}
\frac{\partial^{2} J_{T-1}}{\partial b_{T-1}^{2}}= & -2 \gamma \sigma_{o}^{2} \\
& -2 \gamma \beta \lambda_{T} \frac{\left(\gamma f_{T-1}^{o}-A_{T}+\gamma \lambda_{T}+z_{T-1}\right)^{2}}{\gamma^{2}\left(1-b_{T-1}\right)^{3}} \psi\left(M_{T-1}\right) .
\end{aligned}
$$

Now it is trivial to observe that $\frac{\partial^{2} T_{T-1}}{\partial b_{T-1}^{2}}<0$. Finally, the cross-partial derivative of $J_{T-1}$ with respect to $z_{T-1}$ and $b_{T-1}$ is obtained as:

$\frac{\partial^{2} J_{T-1}}{\partial b_{T-1} \partial z_{T-1}}=-2 \beta \lambda_{T} \frac{\gamma f_{T-1}^{o}-A_{T}+\gamma \lambda_{T}+z_{T-1}}{\gamma\left(1-b_{T-1}\right)^{2}} \psi\left(M_{T-1}\right)$.

Accordingly, the Hessian matrix of $J_{T-1}$ is given by:

$$
\left[\begin{array}{cc}
\frac{\partial^{2} J_{T-1}}{\partial z_{T-1}^{2}} & \frac{\partial^{2} J_{T-1}}{\partial b_{T-1} \partial z_{T-1}} \\
\frac{\partial^{2} J_{T-1}}{\partial b_{T-1} \partial z_{T-1}} & \frac{\partial^{2} J_{T-1}}{\partial b_{T-1}^{2}}
\end{array}\right]=\left[\begin{array}{cc}
A & B \\
C & D
\end{array}\right],
$$

where

$$
\begin{aligned}
A= & -2 \beta \lambda_{T} \frac{1}{\gamma\left(1-b_{T-1}\right)} \psi\left(\frac{A_{T}-\gamma b_{T-1} f_{T-1}^{0}-\gamma \lambda_{T}-z_{T-1}}{\gamma\left(1-b_{T-1}\right)}\right), \\
B= & C=-2 \beta \lambda_{T} \psi\left(\frac{A_{T}-\gamma b_{T-1} f_{T-1}^{0}-\gamma \lambda_{T}-z_{T-1}}{\gamma\left(1-b_{T-1}\right)}\right) \\
& \left(\frac{\gamma f_{T-1}^{o}-A_{T}+\gamma \lambda_{T}+z_{T-1}}{\gamma\left(1-b_{T-1}\right)^{2}}\right), \text { and } \\
D= & -2 \gamma \sigma_{o}^{2}-2 \gamma \beta \lambda_{T} \frac{\left(\gamma f_{T-1}^{o}-A_{T}+\gamma \lambda_{T}+z_{T-1}\right)^{2}}{\gamma^{2}\left(1-b_{T-1}\right)^{3}} \\
& \psi\left(\frac{A_{T}-\gamma b_{T-1} f_{T-1}^{0}-\gamma \lambda_{T}-z_{T-1}}{\gamma\left(1-b_{T-1}\right)}\right) .
\end{aligned}
$$

We have already proven that the first-order principal minors of the Hessian matrix is negative, i.e., $\frac{\partial^{2} T_{T-1}}{\partial z_{T-1}^{2}}<0$ and $\frac{\partial^{2} J_{T-1}}{\partial b_{T-1}^{2}}<0$. In this case, the secondorder principal minor is the determinant of the matrix and is given by

$$
\begin{aligned}
& \frac{\partial^{2} J_{T-1}}{\partial z_{T-1}^{2}} \frac{\partial^{2} J_{T-1}}{\partial b_{T-1}^{2}}-\left(\frac{\partial^{2} J_{T-1}}{\partial b_{T-1} \partial z_{T-1}}\right)^{2} \\
& \quad=4 \gamma \sigma_{o}^{2} \beta \lambda_{T} \frac{1}{\gamma\left(1-b_{T-1}\right)} \psi\left(M_{T-1}\right),
\end{aligned}
$$

which is positive. This concludes that the Hessian is negative definite and $J_{T-1}$ is strictly jointly concave in $z_{T-1}$ and $b_{T-1}$. Then, from concavity preservation under maximization, we conclude that the value function $V_{T-1}$ is also strictly concave in $x_{T-1}$ (see, e.g., Porteus 2002, p. 227).

Now to complete the induction argument, let's assume that the value function $V_{t+1}$ is strictly concave in $x_{t+1}$, and check whether $J_{t}$ is strictly jointly 
concave in $z_{t}$ and $b_{t}$ for $t<T-1$. Recall that

$$
\begin{aligned}
J_{t}\left(x_{t}, z_{t}, b_{t}, \mathcal{P}_{t}\right) & =\left\{\beta E_{\mathcal{P} \mid \mathcal{P}_{t}}^{\mathbb{Q}} \tilde{R}_{t}\left(b_{t}, p_{t+1}\right)-\frac{\left(s_{t}+c_{t}\right)}{a}\left(z_{t}-x_{t}\right)\right. \\
& \left.-r\left(-x_{t}\right)^{+}-h\left(x_{t}\right)^{+}+\beta E_{\mathcal{P} \mid \mathcal{P}_{t}}^{\mathbb{Q}} V_{t+1}\left(x_{t+1}, \mathcal{P}\right)\right\},
\end{aligned}
$$

since $x_{t+1}=z_{t}-d_{t+1}$ is linear in $z_{t}$ and $b_{t}$, if $V_{t+1}$ is strictly concave in $x_{t+1}$, then $V_{t+1}$ is also strictly jointly concave in $z_{t}$ and $b_{t}$. Hence it is sufficient to show that the remaining terms of $J_{t}$, i.e.,

$$
\begin{aligned}
K_{t}= & \beta E_{\mathcal{P} \mid \mathcal{P}_{t}}^{\mathbb{Q}} \tilde{R}_{t}\left(b_{t}, p_{t+1}\right)-\frac{\left(s_{t}+c_{t}\right)}{a}\left(z_{t}-x_{t}\right)-r\left(-x_{t}\right)^{+} \\
& -h\left(x_{t}\right)^{+},
\end{aligned}
$$

are concave. Observing that $\frac{\partial^{2} K_{t}}{\partial z^{2}}=0, \frac{\partial^{2} K_{t}}{\partial b^{2}}=-2 \gamma \sigma_{o}^{2}$ $<0$ and $\frac{\partial^{2} K_{t}}{\partial b_{t} \partial z_{t}}=0$ concludes that $K_{t}$ is concave, and hence $J_{t}$ is strictly jointly concave in $z_{t}$ and $b_{t}$ (note that $V_{t+1}$ is strictly jointly concave). This completes the induction argument.

\section{A.2. Proof of Theorem 1}

PROOF. The first-order condition for $z_{t}$ from Equation (1) is given by

$$
\begin{aligned}
\frac{\partial J_{t}}{\partial z_{t}} & =-\frac{s_{t}+c_{t}}{a}+\beta E_{\mathcal{P} \mid \mathcal{P}_{t}}^{\mathbb{Q}} \frac{\partial V_{t+1}\left(z_{t}-d_{t+1}, \mathcal{P}\right)}{\partial z_{t}}+\mu_{t} \\
& =0, \text { and } \\
& \frac{\partial V_{t}}{\partial x_{t}}=\frac{s_{t}+c_{t}}{a}-h \cdot \mathbf{1}_{\left\{x_{t}>0\right\}}+r .1_{\left\{x_{t}<0\right\}}-\mu_{t},
\end{aligned}
$$

where $\mu_{t}$ is the Lagrange multiplier for $z_{t} \geq x_{t}$. Combining Equations (A1) and (A2) gives

$$
\begin{aligned}
\frac{\partial J_{t}}{\partial z_{t}}= & -\frac{s_{t}+c_{t}}{a}+\beta E_{\mathcal{P} \mid \mathcal{P}_{t}}^{\mathbb{Q}}\left[\frac{s_{t+1}+c_{t+1}}{a}\right. \\
& -h . \mathbf{1}_{\left\{z_{t}>A_{t+1}-\gamma\left(b_{t} f_{t}^{0}+\left(1-b_{t}\right) p_{t+1}+\lambda_{t+1}\right)\right\}} \\
& \left.+r . \mathbf{1}_{\left\{z_{t}<A_{t+1}-\gamma\left(b_{t} f_{t}^{o}+\left(1-b_{t}\right) p_{t+1}+\lambda_{t+1}\right)\right\}}-\mu_{t+1}\right]+\mu_{t}=0 .
\end{aligned}
$$

For ease of exposition, we assume that the discounted processing cost is fixed, i.e., $c_{t}=\beta c_{t+1}$ for $t=1, \ldots, T-2$. Ignoring the constraint $0 \leq b_{t} \leq 1$, the first-order condition for $b_{t}$ from Equation (1) is given by

$$
\begin{aligned}
\frac{\partial J_{t}}{\partial b_{t}}= & \beta E_{\mathcal{P} \mid \mathcal{P}_{t}}^{\mathbb{Q}}\left[\left(A_{t+1}-2 \gamma W_{t+1}\left(b_{t}, p_{t+1}\right)\right)\left(f_{t}^{o}-p_{t+1}\right)\right] \\
& -\beta E_{\mathcal{P} \mid \mathcal{P}_{t}}^{\mathbb{Q}}\left[\frac{\partial V_{t+1}\left(z_{t}-d_{t+1}, \mathcal{P}\right)}{\partial\left(z_{t}-d_{t+1}\right)}\left(\frac{\partial d_{t+1}}{\partial b_{t}}\right)\right]=0 .
\end{aligned}
$$

We know that $\frac{\partial d_{t+1}}{\partial b_{t}}=-\gamma\left(f_{t}^{o}-p_{t+1}\right)$; substituting it in Equation (A4) results in the following myopic condition:

$$
\frac{\partial J_{t}}{\partial b_{t}}=2 \gamma \beta\left(1-b_{t}\right) \sigma_{o}^{2}-\gamma \beta \operatorname{Cov}\left(s_{t+1}, p_{t+1}\right) / a-\gamma \beta u_{t}\left(z_{t}, b_{t}\right)=0
$$

where $\quad u_{t}\left(z_{t}, b_{t}\right)=-r \int_{0}^{M_{t}}\left(f_{t}^{o}-p_{t+1}\right) \psi(p) d p+h \int_{M_{t}}^{\infty}$ $\left(f_{t}^{o}-p_{t+1}\right) \psi(p) d p$ and $M_{t}=\frac{A_{t+1}-\gamma b_{t} f_{t}^{-}-\gamma \lambda_{t+1}-z_{t}}{\gamma\left(1-b_{t}\right)}$.

(a) If $\operatorname{Cov}\left(s_{t+1}, p_{t+1}\right)>0$, then from Equation (A5), it can be easily shown that $b_{t}^{*}<1$.

Ignoring $\mu_{t}$ and $\mu_{t+1}$, and substituting $E_{\mathcal{P} \mid \mathcal{P}_{t}}^{\mathbb{Q}}$ $\left[s_{t+1}\right]=f_{t}^{i}$ in Equation (A3), we obtain $z_{t}^{*}$ as a solution to Equation (2) for $t=1, \ldots, T-2$. Similarly, we can show that Equation (3) follows from Equation (A5) for $t=1, \ldots, T-2$. Also, we can obtain $z_{T-1}^{*}$ and $b_{T-1}^{*}$ for period $T-1$ by substituting $r=p_{T}+\lambda_{T}$ and $h=\lambda_{T}-p_{T}$ in Equations (A3) and (A4), and ignoring the Lagrange multipliers. If $b_{t}^{*}<0$, then since the objective function is concave, the myopic solution is obtained by setting $b_{t}^{*}=0$ and solving Equation (2). Subsequently, we now prove the optimality of this myopic policy when $0 \leq b_{t} \leq 1$.

We structure the proof by showing that the optimal myopic policy never leads to the violation of constraint $z_{t+1}^{*}>z_{t}^{*}-d_{t+1}$. Let $G_{t}=\frac{s_{t}+\beta h a-\beta f_{t}^{i}+c_{t}-\beta c_{t+1}}{\beta(r+h) a}$, then from Equation (2) and equating $M_{t}=\Psi^{-1}\left(G_{t}\right)$, the constraint $z_{t+1}^{*}>z_{t}^{*}-d_{t+1}$ can be written as the following (note that the problem is myopic by definition for $t=T-1)$ :

$$
\begin{aligned}
& A_{t+2}-\gamma b_{t+1} f_{t+1}^{o}-\gamma\left(1-b_{t+1}\right) \Psi^{-1}\left(G_{t+1}\right)-\gamma \lambda_{t+2} \\
& \quad>\gamma\left(1-b_{t}\right)\left(p_{t+1}-\Psi^{-1}\left(G_{t}\right)\right) .
\end{aligned}
$$

Now, let $\bar{U}+\bar{\lambda}$ be the highest possible output price in the local market. At time $t+2, \frac{A_{t+2}}{2 \gamma}$ is the optimal monopoly price for the firm, which is always greater than the price realized in competitive markets. In what follows, given that the firm is a price taker, i.e., $\frac{A_{t+2}}{2 \gamma} \geq \bar{U}+\bar{\lambda}$, we show Equation (A6) is always satisfied.

The worst-case scenario, which violates the constraint in Equation (A6), is that the RHS is large and the LHS is small. The RHS can achieve maximum by setting $b_{t}=0, \Psi^{-1}\left(G_{t}\right)=0$, and $p_{t+1}=\bar{U}+\bar{\lambda}$, such that RHS $=\gamma(\bar{U}+\bar{\lambda})$. In addition, $\frac{\partial z_{t+1}^{*}}{\partial b_{t+1}}=\left(\Psi^{-1}\left(G_{t+1}\right)\right.$ $\left.-f_{t+1}^{o}\right) \gamma$, hence, $z_{t+1}^{*}$ (i.e., the LHS of A6) is monotonically either increasing or decreasing in $b_{t+1}$. If $z_{t+1}^{*}$ is decreasing in $b_{t+1}$, then after setting $b_{t+1}=1$, Equation (A6) becomes $A_{t+2} \geq \gamma f_{t+1}^{o}+\gamma \lambda_{t+2}+\gamma(\bar{U}+\bar{\lambda})$. On the other hand, if $z_{t+1}^{*}$ is increasing in $b_{t+1}$, then after setting $b_{t+1}=0$, Equation (A6) becomes $A_{t+2} \geq \gamma \Psi^{-1}$ $\left[G_{t+1}\right]+\gamma \lambda_{t+2}+\gamma(\bar{U}+\bar{\lambda})$. Finally, note that by construction, $\bar{U}+\bar{\lambda}$ is greater than both $f_{t+1}^{o}+\lambda_{t+2}$ and $\Psi^{-1}\left[G_{t+1}\right]+\lambda_{t+2}$. Now it is easy to observe that, since 
$A_{t+2} \geq 2 \gamma(\bar{U}+\bar{\lambda})$, Equation (A6) is satisfied in both cases.

(b) if $\operatorname{Cov}\left(s_{t+1}, p_{t+1}\right)<0$, then at $b_{t}=1$ we get $\frac{\partial J_{t}}{\partial b_{t}}>0$ from Equation (A5). Since $b_{t} \in[0,1]$ and the objective function is concave, this implies that $b_{t}^{*}=1$. Note that at $b_{t}^{*}=1$ the derivative $\frac{\partial J_{t}}{\partial z_{t}}$ in Equation (A3) does not exist due to the kinks in the objective function. Therefore, the optimality condition has to satisfy $0 \epsilon \frac{\partial J_{t}}{\partial z_{t}}$ as explained in Rockafellar (1970). Observing that $\frac{\partial J_{t}}{\partial z_{t}}=-\frac{s_{t}+c_{t}}{a}+\mu_{t}+\beta E_{\mathcal{P} \mid \mathcal{P}_{t}}^{\mathbb{Q}}\left\{\frac{s_{t+1}+c_{t+1}}{a}-\mu_{t+1}\right\}+$ $\beta\left[r . \mathbf{1}_{\left\{z_{t}<A_{t+1}-\gamma\left(f_{t}^{o}+\lambda_{t+1}\right)\right\}},-h \cdot \mathbf{1}_{\left\{z_{t}>A_{t+1}-\gamma\left(f_{t}^{o}+\lambda_{t+1}\right)\right\}}\right]$ at $b_{t}=$ 1 , now there are two possible cases:

(i) $s_{t}-\beta f_{t}^{i} \leq \beta r a$ : In this case, $z_{t}^{*}=A_{t}-\gamma f_{t}^{o}-$ $\gamma \lambda_{t+1}$ is an optimal solution and myopic indeed. Notice, at $z_{t}^{*}=A_{t}-\gamma f_{t}^{0}-\gamma \lambda_{t+1}$ the leftover inventory is zero; therefore, $\beta E_{\mathcal{P} \mid \mathcal{P}_{t}}^{\mathbb{Q}}\left[\mu_{t+1}\right]=0$.

(ii) $s_{t}-\beta f_{t}^{i}>\beta r a$ : In this case, $\mu_{t}>0$, such that $z_{t}^{*}=x_{t}$ is an optimal solution and myopic indeed.

\section{A.3. Proof of Lemma 3}

Proof. Immediately follows from the concavity of function $R_{t}$ in $p_{t+1}$, and $f_{t}^{0}=\beta E_{\mathcal{P} \mid \mathcal{P}_{t}}^{\mathbb{Q}}\left[p_{t+1}\right]$.

\section{A.4. Proof of Corollary 1}

Proof. From Lemma 2 we know that Equation (3) has a unique solution. Then, if $\operatorname{Cov}\left(p_{t+1}, s_{t+1}\right)>0$, from Equation (A5) it implies that $\frac{\partial J_{t}}{\partial b_{t}}<0$ at $b_{t}=1$. This concludes that $b_{t}^{*}<1$. If $\operatorname{Cov}\left(p_{t+1}, s_{t+1}\right)=0$, from Equation (A5) it implies that $\frac{\partial J_{t}}{\partial b_{t}}=0$ at $b_{t}=1$.

\section{A.5. Proof of Theorem 2}

Proof. From Lemma 3 and Equation (1), we can easily show that $V_{t}\left(x_{t}, \mathcal{P}_{t}\right) \leq \bar{V}_{t}\left(x_{t}, \mathcal{P}_{t}\right)$. From Corollary 1 , we know that $b_{t}^{*}<1$, and hence by the definition of optimality it follows that $V_{t}\left(x_{t}, \mathcal{P}_{t}\right) \leq \bar{V}_{t}\left(x_{t}, \mathcal{P}_{t}\right)$ $<V_{t}\left(x_{t}, \mathcal{P}_{t}\right)$.

\section{A.6. Proof of Theorem 3}

Proof. Since $J_{t}$ is concave in $z_{t}$ for a given $b_{t}$ and $\mathcal{P}_{t}$, the base-stock policy is optimal. If $h<h^{\prime} / a$, then it is possible that $s_{t}+h a-\beta f_{t}^{i} \leq 0$. In this case, from Equation (A3), for $c_{t}=\beta c_{t+1}$ and $b_{t}=1$, it implies that $\beta E_{\mathcal{P} \mid \mathcal{P}_{t}}^{\mathbb{Q}}\left[\mu_{t+1}\right]>0$. This results in the possibility of constraint $z_{t+1} \geq x_{t+1}$ to be binding such that the myopic policy is not optimal.

\section{A.7. Proof of Proposition 1}

Proof. (a) When $\operatorname{Cov}\left(p_{t+1}, s_{t+1}\right)>0$, using Equation (A3) and Corollary 1, we obtain $\frac{\partial^{2} J_{t}}{\partial z_{t} \partial \gamma}=-(r+h)$ $\frac{\left(A_{t+1}-z_{t}\right)}{\gamma^{2}\left(1-b_{t}\right)} \psi\left(M_{t}\right)<0$ and $b_{t}^{*}<1$. Note that $A_{t+1}-z_{t}>0$.
This, in conjunction with the concavity of $J_{t}$ in $z_{t}$, results in $z_{t}^{*}$ decreasing in $\gamma$. When $\operatorname{Cov}\left(p_{t+1}, s_{t+1}\right) \leq 0$, from Theorem $1, z_{t}^{*}$ is non-increasing in $\gamma$.

(b) When $\operatorname{Cov}\left(p_{t+1}, s_{t+1}\right)>0$, from Equation (A5) and Corollary 1, we get

$$
\begin{gathered}
\frac{\partial^{2} J_{t}}{\partial b_{t} \partial \gamma}=\left(\frac{A_{t+1}-\gamma f_{t}^{o}-\gamma \lambda_{t+1}-z_{t}}{1-b_{t}}\right)\left(\frac{A_{t+1}-z_{t}}{\gamma\left(1-b_{t}\right)}\right)(r+h) \\
\text { and } b_{t}^{*}<1 .
\end{gathered}
$$

Then $\frac{\partial^{2} t_{t}}{\partial b_{t} \partial \gamma}>0$ when $z_{t}<z_{t}^{G}=A_{t+1}-\gamma f_{t}^{o}-\gamma \lambda_{t+1}$, and $\frac{\partial^{2} J_{t}}{\partial b_{t} \partial \gamma}<0$ when $z_{t}>z_{t}^{G}$. This, in conjunction with the concavity of $J_{t}$ in $b_{t}$, shows that $b_{t}^{*}$ increases (decreases) with $\gamma$ when $z_{t}<z_{t}^{G}\left(z_{t}>z_{t}^{G}\right)$. When Cov $\left(p_{t+1}, s_{t+1}\right) \leq 0$, then from Theorem $1, b_{t}^{*}$ is always one.

(c) When $\operatorname{Cov}\left(p_{t+1}, s_{t+1}\right)>0$, from Equation (A5) and Corollary 1, we can show $\frac{\partial^{2} J_{t}}{\partial b_{t} \partial r}=\gamma \int_{0}^{M_{t}}\left(f_{t}^{0}-p_{t+1}\right)$ $\psi(p) d p>0$ and $b_{t}^{*}<1$ since by definition $\int_{0}^{M_{t}}$ $\left(f_{t}^{0}-p_{t+1}\right) \psi(p) d p>0$. Also, using Equation (A5) we obtain $\frac{\partial^{2} J_{t}}{\partial b_{t} \partial h}=-\gamma \int_{M_{t}}^{\infty}\left(f_{t}^{0}-p_{t+1}\right) \psi(p) d p>0$. This, in conjunction with the concavity of $J_{t}$ in $b_{t}$, proves the desired result. When $\operatorname{Cov}\left(p_{t+1}, s_{t+1}\right) \leq 0$, then $b_{t}^{*}$ is always one.

(d) When $\operatorname{Cov}\left(p_{t+1}, s_{t+1}\right)>0$, we can show that $\frac{\partial^{2} J_{t}}{\partial z_{t} \partial r}=\beta E_{\mathcal{P} \mid \mathcal{P}_{t}}^{\mathbb{Q}}\left[\mathbf{1}_{\left\{z_{t}<A_{t+1}-\gamma\left(b_{t} f_{t}^{o}+\left(1-b_{t}\right) p_{t+1}+\lambda_{t+1}\right)\right\}}\right]>0, \frac{\partial^{2} J_{t}}{\partial z_{t} \partial h}=$ $\beta E_{\mathcal{P} \mid \mathcal{P}_{t}}^{\mathbb{Q}}\left[-\mathbf{1}_{\left\{z_{t}>A_{t+1}-\gamma\left(b_{t} f_{t}^{o}+\left(1-b_{t}\right) p_{t+1}+\lambda_{t+1}\right)\right\}}\right]<0$ and $b_{t}^{*}<1$. This, in conjunction with the concavity of $J_{t}$ in $z_{t}$, proves the desired result. When $\operatorname{Cov}\left(p_{t+1}, s_{t+1}\right) \leq 0$, from Theorem $1, z_{t}^{*}$ is a constraint with respect to $r$ and $h$.

\section{A.8. Proof of Theorem 4}

Proof. We can construct a similar proof as in Lemma 1 to show that $\hat{V}_{t+1}\left((a+\epsilon) y_{t}+x_{t}-d_{t+1}, \mathcal{P}\right)$ is concave in $y_{t}$. Similar to Lemma 2 , we can establish the joint concavity of the objective function in (4), which establishes that there exists a unique solution for the firstorder conditions (5) and (6), and that the base-stock policy is optimal.

\section{A.9. Proof of Theorem 5}

PROOF. From Equation (4), we approximate the derivative of the value function $\hat{V}_{t}$ with respect to the current inventory $x_{t}$ as $\frac{\partial \hat{V}_{t}}{\partial x_{t}}=\frac{s_{t}+c_{t}}{a}-h \mathbf{1}_{\left\{x_{t}>0\right\}}+r \mathbf{1}_{\left\{x_{t}<0\right\}}$. Thereafter,

$$
\begin{aligned}
\frac{\partial \hat{J}_{t}}{\partial \hat{z}_{t}}= & -\frac{s_{t}+c_{t}}{a}+\beta E_{\epsilon} E_{\mathcal{P} \mid \mathcal{P}_{t}}^{\mathbb{Q}} \frac{\partial \hat{V}_{t+1}\left(x_{t+1}, \mathcal{P}\right)}{\partial x_{t+1}}\left(1+\frac{\epsilon}{a}\right) \\
& =-\frac{s_{t}+c_{t}}{a}+\beta\left\{\frac{f_{t}^{i}+c_{t+1}}{a}-h\right. \\
& \left.+(r+h) \iint_{0}^{\hat{M}_{t}}\left(1+\frac{\epsilon}{a}\right) \psi(p) \phi(\epsilon) d p d \epsilon\right\},
\end{aligned}
$$

where $\hat{M}_{t}=\frac{A_{t+1}-\gamma b_{t} f_{t}^{0}-\gamma \lambda_{t+1}-\hat{z}_{t}-\epsilon\left(\hat{z}_{t}-x_{t}\right) / a}{\gamma\left(1-b_{t}\right)}$. 
Equating (A7) to zero, and defining $\Gamma\left(\hat{M}_{t}\right)=\int\left(1+\frac{\epsilon}{a}\right) \Psi\left(\hat{M}_{t}\right) \phi(\epsilon) d \epsilon$, we get $\Gamma\left(\hat{M}_{t}\right)=$ $\frac{s_{t}+a \beta h-\beta f_{t}^{i}+c_{t}-\beta c_{t+1}}{a \beta(r+h)}$. Similarly, ignoring the constraint $0 \leq b_{t} \leq 1$, we obtain:

$$
\begin{aligned}
\frac{\partial \hat{J}_{t}}{\partial b_{t}}= & 2 \beta \gamma \sigma_{o}^{2}\left(1-b_{t}\right)+\beta \gamma E_{\epsilon} E_{\mathcal{P} \mid \mathcal{P}_{t}}^{\mathbb{Q}} \frac{\partial \hat{V}\left(x_{t+1}, \mathcal{P}\right)}{\partial x_{t+1}}\left(f_{t}^{o}-p_{t+1}\right) \\
= & 2 \beta \gamma \sigma_{o}^{2}\left(1-b_{t}\right)+\beta \gamma E_{\mathcal{P} \mid \mathcal{P}_{t}}^{\mathbb{Q}}\left[\frac{S_{t+1}+c_{t+1}}{a}\left(f_{t}^{o}-p_{t+1}\right)\right] \\
& -\beta \gamma \int\left[-r \int_{0}^{\hat{M}_{t}}\left(f_{t}^{o}-p_{t+1}\right) \psi(p) d p\right. \\
& \left.+h \int_{\hat{M}_{t}}^{\infty}\left(f_{t}^{o}-p_{t+1}\right) \psi(p) d p\right] \phi(\epsilon) d \epsilon \\
= & 2 \beta \gamma \sigma_{o}^{2}\left(1-b_{t}\right)-\beta \gamma \operatorname{Cov}\left(s_{t+1}, p_{t+1}\right) / a \\
& -\beta \gamma \int_{[-r}\left[-\int_{0}^{\hat{M}_{t}}\left(f_{t}^{o}-p_{t+1}\right) \psi(p) d p\right. \\
& \left.+h \int_{\hat{M}_{t}}^{\infty}\left(f_{t}^{o}-p_{t+1}\right) \psi(p) d p\right] \phi(\epsilon) d \epsilon
\end{aligned}
$$

As a result, defining $g_{t}\left(\hat{z}_{t}, b_{t}\right)=\int\left[-r \int_{0}^{\hat{M}_{t}}\left(f_{t}^{o}-\right.\right.$ $\left.\left.p_{t+1}\right) \psi(p) d p+h \int_{\hat{M}_{t}}^{\infty}\left(f_{t}^{o}-p_{t+1}\right) \psi(p) d p\right] \phi(\epsilon) d \epsilon$, and setting $\frac{\partial \hat{\jmath}_{t}}{\partial b_{t}}=0$, we get $2 \sigma_{o}^{2}\left(1-b_{t}\right)=\operatorname{Cov}\left(s_{t+1}, p_{t+1}\right) /$ $a+g\left(\hat{z}_{t}, b_{t}\right)$. Now, solving this equation together with equation (A7) $=0$ gives the unconstrained solutions $\bar{b}_{t}$ and $\bar{z}_{t}$. Since the objective function is concave, the optimal policy is given by (i) if $\bar{b}_{t} \in[0,1]$ then $\hat{z}_{t}^{*}=\bar{z}_{t}$ and $\hat{b}_{t}^{*}=\bar{b}_{t}$ and (ii) if $\bar{b}_{t} \notin[0,1]$ then $\hat{b}_{t}^{*}=\max \left\{\min \left\{\bar{b}_{t}, 1\right\}, 0\right\}$, and $\hat{z}_{t}^{*}$ is obtained by solving Equation $(\mathrm{A} 7)=0$ for $\hat{b}_{t}^{*}=\max \left\{\min \left\{\bar{b}_{t}, 1\right\}, 0\right\}$. Solutions for $t=T-1$ can be obtained analogously.

\section{Appendix B. Value-Neutrality of Hedging Input Procurement}

In this appendix, we provide a mathematical proof of the value-neutrality of input hedging. In addition to the original modeling setup in the manuscript, suppose that the firm may also buy forward contracts for the delivery of the input commodity to reduce its exposure to input price risk. In this case, the timeline of events and decisions in the original manuscript will change as follows (below we only list the changes due to forward buying of the input commodity as the rest of the events and decisions are identical to the ones in the original manuscript):

- At time $t$, the firm decides the volume of input forward contracts, $q_{t}$, to be purchased for delivery at time $t+1$, in addition to other existing decision variables. Let $f_{t, t+1}^{i}$ denote the forward price of the input commodity agreed at time $t$ to be delivered at time $t+1$.

- At time $t$, the firm starts with $x_{t}=z_{t-1}-d_{t}$ units of output inventory and $q_{t-1}$ units of input inventory to be delivered at time $t$ through the input forward agreement engaged in at time $t-1$.

- The firm then decides the echelon output inventory level $z_{t}$ by processing the existing input (from the forward contract) and using the spot market to buy additional input commodity if needed. In particular, $\left(\frac{z_{t}-x_{t}}{a}-q_{t-1}\right)^{+}$ denotes the additional input purchased from the spot market and $\left(q_{t-1}-\frac{z_{t}-x_{t}}{a}\right)^{+}$is the excess input after processing. Note that one unit of input converts to $a$ units of output, and hence $\frac{z_{t}-x_{t}}{a}$ gives how much input is processed.

Before we present the revised mathematical model, below we first show an important lemma that simplifies the exposition of the model.

LEMMA B1. It is suboptimal to carry unprocessed input commodity, i.e., it is optimal to sell unprocessed input commodity $\left(q_{t-1}-\frac{z_{t}-x_{t}}{a}\right)^{+}$in the spot market at the end of each period $t$.

Proof. The proof follows from our no-arbitrage assumption in commodity markets. Suppose at time $t$, the firm owns one unit of input commodity. Now, consider that the firm does not process it and carries it to the next period (by paying a holding cost of $h^{\prime}$ ) with the expectation of using it in the future. Alternatively, at time $t$, the firm may sell the commodity in the spot market and buy a forward contract for the delivery of one unit of input commodity next period. The cost of this alternative strategy is $\beta f_{t, t+1}^{i}-s_{t}$, where $\beta$ is the one period risk-free discount rate. Note that both strategies provide the firm with one unit of input commodity next period. The first one costs $h^{\prime}$ and the second one costs $\beta f_{t, t+1}^{i}-s_{t}$. When we take the difference between the cost of the first and second strategies, we obtain $s_{t}+h^{\prime}-\beta f_{t, t+1}^{i}$, which is called the marginal convenience yield (Goel and Gutierrez 2011, Williams and Wright 1991), and this quantity should always be non-negative; otherwise, it implies arbitrage. In particular, when $s_{t}+h^{\prime}-\beta f_{t, t+1}^{i}<0$, investors may simultaneously long the physical commodity and short the contract and lock in a risk-free profit. This situation is known as cash-and-carry arbitrage in commodity markets (see, e.g., Geman 2005, Ch. 2.5). As a result, the no-arbitrage assumption leads to the fact that carrying unprocessed input is suboptimal. That is, it is optimal to clear the excess input at time $t,\left(q_{t-1}-\frac{z_{t}-x_{t}}{a}\right)^{+}$in the 
spot market. If needed, the firm can buy the same amount in the forward market and avoid paying the marginal convenience yield.

The no-arbitrage assumption and the resulting Lemma B1 are important to prove the value neutrality of input hedging. Using Lemma B1, now the revised optimization model with forward and spot procurement of the input commodity reads as follows:

$$
\begin{gathered}
V_{t}\left(x_{t}, q_{t-1}, \mathcal{P}_{t}\right)=\max _{z_{t}, b_{t}, q_{t} \in \mathcal{A}_{t}\left(x_{t}\right)} J_{t}\left(z_{t}, b_{t}, q_{t}, x_{t}, q_{t-1}, \mathcal{P}_{t}\right) \\
J_{t}\left(z_{t}, b_{t}, q_{t}, x_{t}, q_{t-1}, \mathcal{P}_{t}\right)=\left\{\beta E_{\mathcal{P} \mid \mathcal{P}_{t}}^{\mathbb{Q}} \tilde{R}_{t}\left(b_{t}, p_{t+1}\right)-\beta f_{t, t+1}^{i} q_{t}\right. \\
-s_{t}\left(\frac{z_{t}-x_{t}}{a}-q_{t-1}\right)^{+}+s_{t}\left(q_{t-1}-\frac{z_{t}-x_{t}}{a}\right)^{+} \\
-\frac{c_{t}}{a}\left(z_{t}-x_{t}\right)-r\left(-x_{t}\right)^{+}-h\left(x_{t}\right)^{+} \\
\left.\quad+\beta E_{\mathcal{P} \mid \mathcal{P}_{t}}^{\mathbb{Q}} V_{t+1}\left(z_{t}-d_{t+1}, q_{t}, \mathcal{P}\right)\right\}
\end{gathered}
$$

where, $\mathcal{A}_{t}\left(x_{t}\right)=\left\{z_{t} \geq x_{t}, 0 \leq b_{t} \leq 1\right\}$, and $V_{T}\left(x_{T}\right.$, $\left.q_{T-1}, \mathcal{P}_{T}\right)=s_{T} q_{T-1}+\left(p_{T}-\lambda_{T}\right)\left(x_{T}\right)^{+}-\left(p_{T}+\lambda_{T}\right)\left(-x_{T}\right)^{+}$.

The first term in the objective function, $\beta E_{\mathcal{P} \mid \mathcal{P}_{t}}^{\mathbb{Q}} \tilde{R}_{t}\left(b_{t}, p_{t+1}\right)$, represents the expected revenue from the sales of the output commodity. The second term, $f_{t, t+1}^{i} q_{t}$, gives the cost of forward procurement. The third and fourth terms, $s_{t}\left(\frac{z_{t}-x_{t}}{a}-q_{t-1}\right)^{+}$and $s_{t}\left(q_{t-1}-\frac{z_{t}-x_{t}}{a}\right)^{+}$, are the cost of spot procurement and the revenue from the spot sales of the input commodity, respectively. The fifth term, $\frac{c_{t}}{a}\left(z_{t}-x_{t}\right)$, is the processing cost. The remaining terms are identical to the ones in the original paper, except that the state space of the cost-to-go function now also includes the forward input commodity position $q_{t}$. The following lemma describes the value neutrality of input hedging:

LEMMA B2. Forward procurement of input commodity is value-neutral.

Proof. For $t=1, \ldots, T-2$, we can write the FOC for $q_{t}$ as the following:

$$
\begin{aligned}
& \frac{\partial J_{t}\left(x_{t}, z_{t}, b_{t}, \mathcal{P}_{t}, q_{t}\right)}{\partial q_{t}}=-\beta f_{t, t+1}^{i}+\frac{\partial \beta E_{\mathcal{P} \mid \mathcal{P}_{t}}^{\mathbb{Q}} V_{t+1}\left(z_{t}-d_{t+1}, q_{t}, \mathcal{P}\right)}{\partial q_{t}}=0, \\
& \frac{\partial J_{t}\left(x_{t}, z_{t}, b_{t}, \mathcal{P}_{t}, q_{t}\right)}{\partial q_{t}}=-\beta f_{t, t+1}^{i} \\
& +\frac{\partial \beta E_{\mathcal{P} \mid \mathcal{P}_{t}}^{\mathbb{Q}}\left\{-s_{t+1}\left(\frac{z_{t+1}-x_{t+1}}{a}-q_{t}\right)^{+}+s_{t+1}\left(q_{t}-\frac{z_{t+1}-x_{t+1}}{a}\right)^{+}\right\}}{\partial q_{t}}=0,
\end{aligned}
$$

observing that, in this case, the order of expectation and derivative can be interchanged and using some algebra we obtain:

$$
\begin{aligned}
& \frac{\partial J_{t}\left(x_{t}, z_{t}, b_{t}, \mathcal{P}_{t}, q_{t}\right)}{\partial q_{t}}=-\beta f_{t, t+1}^{i}+\beta E_{\mathcal{P} \mid \mathcal{P}_{t}}^{\mathbb{Q}}\left[s_{t+1} \mathcal{I}_{\left\{d_{t+1} \geq z_{t}+a q_{t}-z_{t+1}\right\}}\right] \\
& +\beta E_{\mathcal{P} \mid \mathcal{P}_{t}}^{\mathbb{Q}}\left[s_{t+1} \mathcal{I}_{\left\{d_{t+1} \leq z_{t}+a q_{t}-z_{t+1}\right\}}\right]=0 \\
& \frac{\partial J_{t}\left(x_{t}, z_{t}, b_{t}, \mathcal{P}_{t}, q_{t}\right)}{\partial q_{t}}=-\beta f_{t, t+1}^{i}+\beta E_{\mathcal{P} \mid \mathcal{P}_{t}}^{\mathbb{Q}}\left[s_{t+1} \mathcal{I}_{\left\{\hat{p}_{t+1} \geq p_{t+1}\right\}}\right] \\
& +\beta E_{\mathcal{P} \mid \mathcal{P}_{t}}^{\mathbb{Q}}\left[s_{t+1} \mathcal{I}_{\left\{\hat{p}_{t+1} \leq p_{t+1}\right\}}\right]=0
\end{aligned}
$$

where $\hat{p}_{t+1}=\frac{A_{t+1}-\left(z_{t}+a q_{t}-z_{t+1}\right)}{\gamma\left(1-b_{t}\right)}-\frac{\left(\lambda_{t+1}+b_{t} f_{t}^{o}\right)}{\left(1-b_{t}\right)}$. Then,

$$
\begin{aligned}
\frac{\partial J_{t}\left(x_{t}, z_{t}, b_{t}, \mathcal{P}_{t}, q_{t}\right)}{\partial q_{t}}= & -\beta f_{t, t+1}^{i}+\beta E_{\mathcal{P} \mid \mathcal{P}_{t}}^{\mathbb{Q}}\left[s _ { t + 1 } \left(\mathcal{I}_{\left\{\hat{p}_{t+1} \geq p_{t+1}\right\}}\right.\right. \\
& \left.+\mathcal{I}_{\left\{\hat{p}_{t+1} \leq p_{t+1}\right\}}\right]=0 \\
\frac{\partial J_{t}\left(x_{t}, z_{t}, b_{t}, \mathcal{P}_{t}, q_{t}\right)}{\partial q_{t}}= & -\beta f_{t, t+1}^{i}+\beta E_{\mathcal{P} \mid \mathcal{P}_{t}}^{\mathbb{Q}}\left[s_{t+1}\right]=0 .
\end{aligned}
$$

Finally, note that the no-arbitrage assumption also requires that the contracts are fairly priced, i.e., $f_{t, t+1}^{i}=E_{\mathcal{P} \mid \mathcal{P}_{t}}^{\mathbb{Q}}\left[s_{t+1}\right]$. Hence, it holds that $\frac{\partial J_{t}(\cdot)}{\partial q_{t}}=-\beta f_{t, t+1}^{i}+$ $\beta E_{\mathcal{P} \mid \mathcal{P}_{t}}^{\mathbb{Q}}\left[s_{t+1}\right]=0$ for all $q_{t}$, and the level of forward buying, $q_{t}$, is irrelevant to firm value. ${ }^{8}$ This result may also be generalized to other financial derivatives for trading the input commodity.

\section{Appendix C. The Choice of Demand Parameters}

Economics literature shows that ethanol demand presents strong price elasticity. In particular, empirical research finds that price elasticity of ethanol demand ranges between -0.43 and -2.92 (Elobeid and Tokgoz 2008, Luchansky and Monks 2009, Roberts and Schlenkera 2013). Following these results in the literature, we consider a downward sloping linear demand function for ethanol, i.e., $d_{t+1}=A-\gamma p_{t+1}$. We normalize the firm specific market size to $A=500$ units, and consider three levels of price sensitivity: high $(\gamma=160)$, medium $(\gamma=120)$ and low $(\gamma=80)$. We select these levels of $\gamma$ such that the resulting price elasticities are consistent with the empirical observations. In particular, in our demand model, ethanol price elasticities for the mean price ranges from -0.56 (for $\gamma=80$ ) to $-2.57(\gamma=160)$. Note that our model is tested on futures price data for corn and ethanol from the Chicago Mercantile Exchange between 4/1/2005 and $12 / 31 / 2011$. In this period, the average ethanol price was $\$ 2.25 /$ gallon.

\section{Notes}

${ }^{1}$ According to Van Mieghem (2003), "Mitigating risk, or hedging, involves taking counterbalancing actions so that, 
loosely speaking, the future value varies less over the possible states of nature. If these counterbalancing actions involve trading financial instruments, including short-selling, futures, options, and other financial derivatives, we call this financial hedging." In our context, we refer to financial hedging as selling the output commodity using a forward contract or an index-based contract.

${ }^{2}$ Perfect hedging is defined as a hedge that completely eliminates price risk.

${ }^{3} \mathrm{~A}$ jobber procures gasoline from the refineries, then processes it with additives in accordance with state and environmental regulations to sell the gasoline to unbranded retailers. ${ }^{4}$ For models with similar assumptions on the correlation between demand and price, see Kazaz (2004) and Secomandi and Kekre (2014).

${ }^{5}$ In the economics literature, Pindyck (2004) defines marginal convenience yield as the economic cost of storage.

${ }^{6}$ In section 5, we illustrate the computational procedure.

${ }^{7}$ For the demand function, we normalize the firm specific market size to $A=500$ units, and consider three levels of price sensitivity: high $(\gamma=160)$, medium $(\gamma=120)$ and low $(\gamma=80)$ (see Appendix $C$ for more details).

${ }^{8}$ The same result may also be shown using a similar line of reasoning for the last decision period $T-1$. For brevity it is omitted here.

\section{References}

Bazerman, M. H., J. J. Gillespie. 1998. Betting on the future: The virtues of contingent contracts. Harv. Bus. Rev. 77(5): 155-160.

Berling, P., V. Martinez-de Albeniz. 2011. Optimal inventory policies when purchase price and demand are stochastic. Oper. Res. 59(1): 109.

Bessembinder, H. 1991. Forward contracts and firm value: Investment incentive and contracting effects. J. Financ. Quant. Anal. 26(4): 519-532.

Biyalogorsky, E., E. Gerstner. 2004. Contingent pricing to reduce price risks. Market. Sci. 23(1): 146-155.

Boyabatli, O., P. Kleindorfer, S. Koontz. 2011. Integrating longterm and short-term contracting in beef supply chains. Management Sci. 57: 1771-1787.

Boyabatli, O., Q. J. D. Nguyen, T. Wang 2016. Capacity management in agricultural commodity processing and application in the palm industry. Working paper, Lee Kong Chian School of Business, Singapore.

Bresnahan, T. F. 1982. The oligopoly solution concept is identified. Econ. Lett. 10(1): 87-92.

Caldentey, R., M. B. Haugh. 2009. Supply contracts with financial hedging. Oper. Res. 57(1): 47-65.

Chen, X., M. Sim, D. Simchi-Levi, P. Sun. 2007. Risk aversion in inventory management. Oper. Res. 55(5): 828.

Chod, J., N. Rudi, J. A. Van Mieghem. 2010. Operational flexibility and financial hedging: Complements or substitutes? Management Sci. 56(6): 1030-1045.

Dahlgran, R. 2010. Ethanol futures: Thin but effective?-why? Proceedings of the NCCC-134 Conference on Applied Commodity Price Analysis, Forecasting, and Market Risk Management. St. Louis, MO.

Deaton, A., G. Laroque. 1992. On the behaviour of commodity prices. Rev. Econ. Stud. 59(1): 1-23.

Devalkar, S., R. Anupindi, A. Sinha. 2011. Integrated optimization of procurement, processing, and trade of commodities. Oper. Res. 59(6): 1369-1381.
Devalkar, S. K., R. Anupindi, A. Sinha 2016. Dynamic risk management of commodity operations: Model and analysis. Working paper, Indian School of Business, India.

Ding, Q., L. Dong, P. Kouvelis. 2007. On the integration of production and financial hedging decisions in global markets. Oper. Res. 55(3): 470-489.

Dong, L., H. Liu. 2007. Equilibrium forward contracts on nonstorable commodities in the presence of market power. Oper. Res. 55(1): 128-145.

Elobeid, A., S. Tokgoz. 2008. Removing distortions in the us ethanol market: What does it imply for the United States and Brazil? Am. J. Agr. Econ. 90(4): 918-932.

Federgruen, A., A. Heching. 1999. Combined pricing and inventory control under uncertainty. Oper. Res., 47(3): 454-475.

Franken, J., J. Parcell. 2003. Cash ethanol cross-hedging opportunities. J. Agric. Appl. Econ. 35(3): 509-516.

Froot, K. A., D. S. Scharfstein, J. C. Stein. 1993. Risk management: Coordinating corporate investment and financing policies. J. Financ. 48(5): 1629-1658.

Gaur, V., S. Seshadri. 2005. Hedging inventory risk through market instruments. Manuf. Serv. Oper. Manag. 7(2): 103-120.

Geman, H. 2005. Commodities and Commodity Derivatives. Modeling and Pricing for Agriculturals, Metals and Energy, Wiley Finance, Chichester (Grande-Bretagne).

Goel, A., G. J. Gutierrez. 2009. Effect of term structure of futures price on the spot procurement policies. Working paper, Case Western Reserve University.

Goel, A., G. J. Gutierrez. 2011. Multiechelon procurement and distribution policies for traded commodities. Management Sci. 57(12): 2228-2244.

Hahn, W., J. Dyer. 2008. Discrete time modeling of mean-reverting stochastic processes for real option valuation. Eur. J. Oper. Res. 184(2): 534-548.

Henig, M., Y. Gerchak. 1990. The structure of periodic review policies in the presence of random yield. Oper. Res. 38(4): 634-643.

Ho, T. 1984. Intertemporal commodity futures hedging and the production decision. J. Financ. 39(2): 351-376.

Inderfurth, K., P. Kelle, R. Kleber. 2014. The effect of material price and product demand correlations on combined sourcing and inventory management. Working paper no. 13/ 2014, Universitat Macdeburg, Faculty of Economics and Management.

Jin, Y., P. Jorion. 2006. Firm value and hedging: Evidence from us oil and gas producers. J. Financ. 61(2): 893-919.

Kamrad, B., P. Ritchken. 1991. Multinomial approximating models for options with k state variables. Management Sci. 37(12): $1640-1652$.

Kazaz, B. 2004. Production planning under yield and demand uncertainty with yield-dependent cost and price. Manuf. Serv. Oper. Manag. 6(3): 209-224.

Kouvelis, P., R. Li, Q. Ding. 2013. Managing storable commodity risks: The role of inventory and financial hedge. Manuf. Serv. Oper. Manag. 15(3): 507-521.

Losq, E. 1982. Hedging with price and output uncertainty. Econ. Lett. 10(1-2): 65-70.

Luchansky, M., J. Monks. 2009. Supply and emand elasticities in the us ethanol fuel market. Energy Econ. 31(3): 403-410.

Modigliani, F., M. Miller. 1958. The cost of capital, corporation finance and the theory of investment. Am. Econ. Rev. 48(3): 261-297.

Moschini, G., H. Lapan. 1992. Hedging price risk with options and futures for the competitive firm with production flexibility. Int. Econ. Rev. 33(3): 607-618. 
Moschini, G., H. Lapan. 1995. The hedging role of options and futures under joint price, basis, and production risk. Int. Econ. Rev. 36(4): 1025-1049.

Noparumpa, T., B. Kazaz, S. Webster. 2015. Wine futures and advance selling under quality uncertainty. Manuf. Serv. Oper. Manag. 17(3): 411-426.

Pindyck, R. 2004. Volatility and commodity price dynamics. J. Futures Market. 24(11): 1029-1047.

Plambeck, E., T. Taylor. 2011. On the flexibility to adapt to cofluctuations in feedstock costs and output prices. Working paper, Stanford University, Stanford.

Plambeck, E. L., T. A. Taylor. 2013. On the value of input efficiency, capacity efficiency, and the flexibility to rebalance them. Manuf. Serv. Oper. Manag. 15(4): 630-639.

Porteus, E. L. 2002. Foundations of Stochastic Inventory Theory. Stanford University Press, Stanford.

Roberts, M. J., W. Schlenkera. 2013. Identifying supply and demand elasticities of agricultural commodities: Implications for the us ethanol mandate. Am. Econ. Rev. 103(6): 2265-2295.

Rockafellar, R. T. 1970. Convex Analysis, Volume 28 of Princeton Mathematics Series. Princeton University Press, Princeton, NJ.

Rolfo, J. 1980. Optimal hedging under price and quantity uncertainty: The case of a cocoa producer. J. Polit. Econ. 88(1): 100-116.

Samuelson, W. 1986. Bidding for contracts. Management Sci. 32(12): 1533-1550.

Schwartz, E. 1997. The stochastic behavior of commodity prices: Implications for valuation and hedging. J. Financ. 52(3): 923-973.
Schwartz, E., J. Smith. 2000. Short-term variations and long-term dynamics in commodity prices. Management Sci. 46(7): 893-911.

Secomandi, N. 2010. Optimal commodity trading with a capacitated storage asset. Management Sci. 56(3): 449-467.

Secomandi, N., S. Kekre. 2014. Optimal energy procurement in spot and forward markets. Manuf. Serv. Oper. Manag. 16(2): 270-282.

Seppi, D. 2002. Risk-neutral stochastic processes for commodity derivative pricing. In E. Ron, ed. Real Options and Energy Management: Using Options Methodology to Enhance Capital Budgeting Decisions, Chapter 2. Risk Publications, London, England.

Smith, C. W., R. M. Stulz. 1985. The determinants of firms' hedging policies. J. Financ. Quant. Anal. 20(04): 391-405.

Sobel, M. J., V. Babich. 2012. Optimality of myopic policies for dynamic lot-sizing problems in serial production lines with random yields and autoregressive demand. Oper. Res. 60(6): 1520-1536.

Turcic, D., P. Kouvelis, E. Bolandifar. 2015. Hedging commodity procurement in a bilateral supply chain. Manuf. Serv. Oper. Manag. 17(2): 221-235.

Van Mieghem, J. A. 2003. Commissioned paper: Capacity management, investment, and hedging: Review and recent developments. Manuf. Serv. Oper. Manag. 5(4): 269-302.

Wakeley, H., C. Hendrickson, W. Griffin, H. Matthews. 2009. Economic and environmental transportation effects of large-scale ethanol production and distribution in the united states. Environ. Sci. Technol. 43(7): 2228-2233.

Williams, J., B. Wright. 1991. Storage and Commodity Markets. Cambridge University Press, New York. 